\title{
Soziale Elternschaft und soziale Zugehörigkeit. Reflexionen der Zürcher Adoptionsstudie
}

\author{
Thomas Gabriel · Samuel Keller
}

Online publiziert: 27. März 2020

(C) Der/die Autor(en) 2020

Zusammenfassung Der Artikel reflektiert den Begriff und das Konzept der ,,Sozialen Elternschaft“" vor dem Hintergrund ausgewählter Befunde der ,Zürcher Adoptionsstudie“. Im Fokus stehen dabei erziehungswissenschaftliche Fragen an Übergänge vom Paar- zum Familienleben, die am empirischen Material verdichtet werden. Anhand des Konzepts der „Leiblichkeit“ wird eine zentrale Herausforderung exemplarisch aufgegriffen und diskutiert. Daran wird deutlich, wie gesellschaftliche Wandlungsprozesse, normative Ansprüche und Familienbilder sowie behördliche Überprüfungen die Suche nach Normalität von „Eltern unter Beobachtung“ beeinflussen. Sie erschweren oft eine eigenständige und sinnstiftende Entwicklung ihrer „Sozialen Elternschaft“, die auch das Kind einschließt.

Schlüsselwörter Adoption · Soziale Elternschaft · Vulnerabilität · Kinder- und Jugendhilfe · Adoptionsforschung

Prof. Dr. T. Gabriel ( $₫) \cdot$ Dr. S. Keller

Departement Soziale Arbeit, Institut für Kindheit, Jugend und Familie, Zürcher Hochschule für Angewandte Wissenschaften, Pfingstweidstrasse 96, 8005 Zürich, Schweiz

E-Mail: thomas.gabriel@zhaw.ch

Dr. S. Keller

E-Mail: samuel.keller@zhaw.ch 


\title{
Social parenthood and social belonging. Reflections on findings of the Zurich Study on Adoption
}

\begin{abstract}
The article reflects the concept of "social parenthood" against the background of selected findings of the "Zurich Adoption Study". The focus is based on educational science questions about transitions from couple to family life, which are examined on the basis of empirical material. Using the concept of "Leiblichkeit" as an example, a central challenge will be taken up and discussed. This makes it clear how social processes of change, normative demands and family images as well as official examinations influence the search for normality of "parents under observation". They often hinder an independent and meaningful development of their "social parenthood", which includes the child as well.
\end{abstract}

Keywords Adoption · Adoption research · Parenting · Vulnerability · Child Care

\section{Soziale Elternschaft und Adoption}

In Adoptivfamilien verdichten sich Fragen des Verhältnisses zwischen sozialer und biologischer Elternschaft in einer einmaligen und analytisch fassbaren Form. In diesem Feld der Adoptivfamilien bietet sich die Möglichkeit, das Konzept „Sozialer Elternschaft" sowohl konzeptionell-begrifflich als auch empirisch zu reflektieren. Dies ist aus erziehungswissenschaftlicher Perspektive interessant, da hier wissenschaftliche Anschauung auf das gelebte Leben in Familien trifft und Widersprüche sowie Herausforderungen analytisch fassbar werden.

Ausgehend von Erkenntnissen aus einer Langzeitstudie mit Adoptivfamilien im Kanton Zürich wird dieser Beitrag deshalb die folgenden Fragen mit erziehungswissenschaftlicher Aussagekraft zu beantworten versuchen:

- Wie kann eine explizierte und nicht-biologische Elternschaft die Handlungs- und Seinssicherheit der Adoptiveltern zwischen Innen- und Außenwahrnehmungen beeinflussen?

- Wie werden Übergangsphasen vom Paar zur Familie unter behördlicher Beobachtung und Prüfung ausgestaltet und begründet?

- Spezifisch: Welche Bedeutung spielt dabei die Aushandlung von Zugehörigkeit und ,Leiblichkeit“"?

Um diese zu beantworten, werden einleitend die zentralen Begrifflichkeiten geklärt und anschließend die Bedeutung des Eignungsverfahrens künftiger Adoptiveltern dargelegt. Danach folgt ein Einblick in das Forschungsdesign der Zürcher Adoptionsstudie, auf deren Ergebnisse vorliegender Beitrag beruht. Die Auswahl der Ergebnisse ist selektiv, der Fokus liegt auf dem Übergang vom Paar zur sozialen Elternschaft. Dabei interessieren insbesondere die intersubjektive Wahrnehmung und Bedeutung von Krisen und Normalitäten sowie die sogenannte „Leiblichkeit“" (Gabriel und Keller 2013, 2018) als ein Schlüsselkonzept. Die abschließende Diskussion widmet sich der empirisch nachgewiesenen Vulnerabilität sozialer Elternschaft 
in Adoptionen in Wechselwirkung zu einer normativen Anfälligkeit des erziehungswissenschaftlichen Diskurses.

Auch wenn sich die Anzahl der Adoptionen in Westeuropa in den vergangenen Jahren stark verringert hat, stellen sowohl die behördliche Praxis des (Eignungs-)Verfahrens für künftige Adoptiveltern als auch die Erfahrungen der Eltern und ihrer Kinder ein Brennglas für aktuelle gesellschaftliche Wandlung und Aushandlung von Familie, Elternschaft und Kindheit dar (vgl. Keller 2018, S. 20). In Adoptionen könnte sich folglich ein allgemeiner gesellschaftlicher Trend verdichten, den Peuckert (2012) als „Entkoppelung von biologischer und sozialer Elternschaft“ benennt (Peuckert 2012, S. 381). Demnach fallen statistisch betrachtet biologische und soziale Elternschaft zunehmend auseinander und Blutsverwandtschaft stellt immer weniger das Kriterium für familiale Lebensgemeinschaften dar. Gleichwohl kann davon ausgegangen werden, dass die Aushandlung einer (neuen) Ordnung der Familie, einer ,neue Normalität“ (Eggen 2018, S. 181) noch im vollen Gange ist und in Bezug auf gesellschaftlich verankerte Erwartungen und Werte nur zäh vorankommt (vgl. z. B. Beck 2014; Bernard 2014).

Um die Tragweite dieser Entwicklung zu verstehen, bedarf es eines kurzen historischen Rückblicks. Der Verbindung von biologischer Elternschaft und bürgerlichem Familienideal wurde in Europa im Zuge der Industrialisierung und Individualisierung des 19. Jahrhunderts eine zentrale Bedeutung zugewiesen: „Familie wird als Kernelement der Nationalstaaten verstanden, womit ihr auch eigenständige Pflichten auferlegt werden. Im Kern hat sie über eine ideale ,Aufzucht' der nachfolgenden Generation für die Erhaltung des Staates zu sorgen“ (Grubenmann 2009, S. 652). Dieser bürgerliche Erziehungsauftrag wird von Rousseau in aufklärerisches Gedankengut eingebettet und bildet bis heute die Grundlage vieler der nachfolgenden staatsphilosophischen und pädagogischen Überlegungen zu Elternschaft (Grubenmann 2009).

Heute tragen zahlenmäßig insbesondere trennungs- oder scheidungsbedingte EinEltern-Familien, Stiefkind- und Patchworkfamilien sowie Familien mit gleichgeschlechtlichen Eltern oder Queer-Familien zum Wandel der Realität familialer Lebensgemeinschaften bei. Zudem sind neue medizinische Formen zur Reproduktion (vgl. etwa Bernard 2014) als ursächlich für den aktuellen Wandel familialer Gefüge zu verstehen, in welchen zunehmend ,die Einheit von Reproduktionstriade und erziehender Familie auseinanderfällt" (Peuckert 2012, S. 381). Hinzu kommt eine nicht zu unterschätzende Anzahl verdeckter Formen fragmentierter Elternschaft, „die dann entsteht, wenn Kinder außerehelich gezeugt werden und als gemeinsame Kinder der Ehepartner aufwachsen“ (Peuckert 2012, S. 381). Bei Adoptivfamilien schließlich - die Verwandtschaftsadoption ausgeschlossen - entsteht Elternschaft ohne biologische Verwandtschaftsverhältnisse zwischen Eltern und Kind (Gabriel und Keller 2013, S. 7f.).

Ausgehend von dieser Ausgangslage im Feld der Adoption wird in diesem Beitrag ,soziale Elternschaft“" zunächst deskriptiv und in Anlehnung an Eggen (2018) als sozial definierte und sozial zugeschriebene Rolle verstanden, die bestimmte Aufgaben der Erziehung sowie die damit verbundene Verantwortung beinhaltet. Dabei teilen oder - wie dies bei Adoptiveltern zutrifft - übernehmen die sozialen Eltern diese Rolle schrittweise vom Staat, vermittelt durch dessen Institutionen. Weiterhin Eggen folgend, wird ,soziale Elternschaft“ analytisch aufgeteilt in rechtliche und 
familiale Elternschaft, wobei - vor allem im Sinne der obigen Fragestellungen - das Verhältnis dieser zwei Dimensionen zueinander hier von Interesse sein wird:

- Rechtliche Elternschaft entsteht - und dies stellt stets einen zentralen Akt der Adoptionsverfahren dar - durch rechtliche Zuordnung eines Kindes zu Person(en), die damit auch Pflichten und Rechte gegenüber dem Kind annehmen muss.

- Familiale Elternschaft entsteht, wenn eine Person die Elternverantwortung für ein Kind durch Selbstverpflichtung übernimmt. Im Sinne der „Familie als Herstellungsleistung“ (Jurczyk 2014, S. 50) entscheiden immer auch die beteiligten erwachsenen Personen über familiale Elternschaft und Anzahl familialer Eltern.

Damit grenzt sich das vorliegende Verständnis sozialer Elternschaft einerseits von biologischer Elternschaft ab, die sich über das biologische Abstammungsverhältnis ergibt - wobei auch hier neue hybride Formen bestehen (sogenannte ,Leihmütter stellen zwar die biologischen, aber nicht die genetischen Mütter dar). Auch unterscheidet sich die soziale von der psychischen Elternschaft, die gemäß Eggen (Jurczyk 2014) durch Gedanken und Gefühle, die jede/r unabhängig von biologischer und genetischer Verwandtschaft individuell bildet - oder bspw. bei postnatalen Depressionen oder unerfüllt bleibendem Kinderwunsch trotz Kind - auch nicht.

Diese komplexen und vielschichtigen Dimensionen der möglichen Definition von (sozialer) Elternschaft machen es nicht nur für die Rechtsprechung in Streitfällen anspruchsvoll (Vaskovics 2016, S. 195). Auch das methodisch geleitete Verstehen familiärer Situationen im Kontext von Kindesschutzfragen (Cinkl und Krause 2014) bspw. einer rekonstruktiven (Familien-)Diagnostik (z. B. Ader et al. 2001; Uhlendorff et al. 2008) oder systemische Familieninterventionen (vgl. bspw. Merritts 2016) sind mit dieser Komplexität konfrontiert. Vor allem aber entstehen in der hier angenommenen Lücke zwischen faktisch bestehender Diversität von Elternschaft und der tatsächlichen Toleranz im Sozialen gegenüber unterschiedlichen Lebensformen große Herausforderungen. Diese Herausforderungen können empirisch über jene fassbar werden, die sich durch Dritte nicht als Eltern oder Familie akzeptiert fühlen (vgl. z. B. Jurczyk 2014). Unterschiedliche Studien verweisen bereits darauf, dass sich eine sozial begründete Verunsicherung insbesondere bei Kindern und Eltern bemerkbar machen kann, die offiziell - und gerade bei internationalen Adoptionen oft auch visuell - keine biologischen Verwandtschaften aufweisen: bei Adoptivfamilien (vgl. Wegar 2004; DJI 2017a; Michaelsen 2017; Gassmann 2018).

Adoptionen stellen gerade auch bezüglich rechtlicher Elternschaft als eine Dimension sozialer Elternschaft (vgl. Eggen 2018) eine Ausnahme dar. Denn diese Dimension von Elternschaft wird in biologischen Familien zumeist erst dann zum Thema, wenn es zu ausgeprägten Krisen zwischen den Eltern, zwischen Eltern und Kind oder zwischen Behörden und Eltern bzw. Familie kommt. Der Gang zu Anwälten und zu Gerichten expliziert dann die Krise und stellt die (Ausprägung von) Elternschaft rechtlich infrage (vgl. Eggen 2018, S. 185). Bei Adoption hingegen wird dieser juristische Gang ex ante vollzogen. Dass in der sogenannten ,Eignungsabklärung“" als Teil des behördlichen Bewilligungsverfahrens zur Adoption die Elternschaft erstmals zu einem Zeitpunkt überprüft wird, an dem zwar ein ausdrücklicher Kindeswunsch eines Paares, jedoch noch kein Kind anwesend ist, macht einen wichtigen Teil der erziehungswissenschaftlichen und auch gesellschaftlichen 
Relevanz dieser Thematik aus (vgl. Gabriel und Keller 2013, S. 25). Das Kind ist zu diesem Zeitpunkt des Verfahrens, ebenso wie die imaginierte Idee von Familie ein Konstrukt der zukünftigen Adoptiveltern, das in der Interaktion mit den Behörden geformt wird. Erst mit der Ankunft des Kindes werden diese Konstrukte im realen Alltag der Familie ihre Tauglichkeit erweisen müssen.

Zudem ergibt sich hier eine wissenschaftlich fachliche Frage: In Anbetracht der multiplen und hybriden Formen von Elternschaft besteht die zentrale Herausforderung des erziehungswissenschaftlichen Diskurses darin, zu definieren, was ,gute“ oder zumindest ,genügende“ - Elternschaft ausmacht, was gelingendes Aufwachsen unter heutigen Bedingungen zentral bedingt. Denn dieser Diskurs muss auch zum Ziel haben mit zu beeinflussen, wie „Eltern unter Beobachtung“ - und dazu gehören alle nicht-biologischen Eltern wie Pflege- oder Adoptiveltern (vgl. Gassmann 2018), aber auch Stiefeltern - von offizieller Seite und der Gesellschaft mit welchen Ansprüchen, Idealen und auch Risiken von Elternschaft konfrontiert werden. Diese spezifische Differenz zwischen biologischer und sozialer Elternschaft führt Gassmann (2018) auf die deutlich höhere Verletzbarkeit bzw. das deutlich höhere Risiko zurück, in der Elternrolle bei ,erworbener Elternschaft“ infrage gestellt zu werden (Gassmann 2018, S. 323).

Deshalb gilt in einem ersten Schritt der Fokus dem Übergang vom Paar zur Elternschaft im Rahmen der sogenannten Eignungsabklärung künftiger Adoptiveltern. Darin interessiert insbesondere, welche Veränderungstendenzen, welche Ansprüche und welche erziehungswissenschaftlichen Fragen an soziale Elternschaft sich hier verdichten.

\section{Wenn Paare staatlich als potenziell gute Eltern anerkannt werden}

Aktuell ist das Adoptionsfeld in Europa vor allem einem steten und „hybriden“ (vgl. Reckwitz 2015) Wandel von Themen wie Fortpflanzungstechniken, Familienbilder und internationaler Ethik unterworfen, die national- und geopolitische Entwicklungen gewissermaßen widerspiegeln (vgl. Bernard 2014). Seit diesem Jahrtausend ist beispielsweise ein kontinuierlicher und starker Rückgang der nationalen und internationalen Adoptionen in Westeuropa und den USA zu verzeichnen. In der Schweiz wie auch in Deutschland ist die Anzahl der Adoptionen seit 1980 um Dreiviertel zurückgegangen.

Für die Schweiz und Deutschland mit hohen Anteilen an internationalen Adoptionen lässt sich der Rückgang einerseits durch deren Ratifizierung des Haager Adoptionsübereinkommens begründen. Damit nahmen die Fürsorgebedürfnisse international adoptierter Kinder einerseits stark zu (vgl. DJI 2017a), vor allem aber schlossen viele Herkunftsländer nach dem Aufdecken organisierter Kinderhandelsstrukturen internationale Adoptionen ganz aus (vgl. Smolin 2005; Ballard et al. 2015; Bitter et al. 2019). Diesen Themen tragen zusätzlich potenzielle Verunsicherungen an die soziale Elternschaft im Rahmen von Adoptionen - sowohl auf rechtlicher als auch auf familialer Dimension von Elternschaft - heran. Zudem machen sich auf Seiten von Paaren mit (unerfülltem) Kinderwunsch nebst der öffentlichen Sensibilisierung auf internationale Problemstellungen wie bspw. Kinderhandel auch weitere 
Wandel bemerkbar. Dazu zählen veränderte Familienbilder und Familienentwürfe, Ausbau der Kinder- und Jugendhilfe und familienpolitischer Leistungen, (vgl. Peuckert 2012, S. 396f.; DJI 2017b, S. 7 f.) sowie Fortschritte in der Reproduktionsmedizin und weiterer Alternativen des „Kinder Machens“ (vgl. Bernard 2014).

In den Erfahrungen und Imaginationen von Eltern - und insbesondere von Adoptiveltern - scheint die latent nach wie vor wirkmächtige Ideologie der biologischen, sogenannten Kernfamilie angesichts der Tatsache einer enormen Diversifizierung gelebter Elternschaft zunehmend paradox. Dies spiegelt sich nicht nur im behördlichen Handeln, sondern auch im Erleben der Eltern, die adoptieren, wider. Als Bild hierfür kann eine Mutter gelten, die als einen der Gründe für die Adoption angibt, dass sie im Fall einer Adoption, ,wie andere Eltern auch am Sonntag spazieren gehen kann". ${ }^{1}$ Dies verweist auf eine Paradoxie gesellschaftlicher Normen am sozialen Ort des Sonntagsspaziergangs, den bereits Heinrich Heine (1827, S. 141) in seiner bürgerlichen Konstruktion ironisch und bildhaft beschrieben hat. Der Sonntagsspaziergang ist hier der soziale Ort, an dem familiäre Normalität rekursiv durch das Handeln hergestellt und durch die Nachbarschaft bezeugt werden kann. Doch könnte genau der Wandel hin zu multiplen Formen der Elternschaft selbst die Ursache für ein zunehmendes Festhalten an einem eindeutigen, statischen Eltern- und Familienbild darstellen. Eindeutige Orientierungen erwecken zumindest den Anschein, den Verunsicherungen durch die multiplen Deutungsangebote entgegenwirken zu können. Dass jedoch gerade dieses Festhalten an bürgerlichen, vornehmlich biologistisch begründeten Familienidealen die Paradoxien im Erleben der Adoptiveltern verstärkt und zu neuen Verunsicherungen führt, belegt das empirische Material der Zürcher Adoptionsstudie (Gabriel und Keller 2013), deren Design und ausgewählte Ergebnisse später noch vorgestellt werden.

Angesichts dieser Kontextualisierung von sozialer Elternschaft und Adoption kann hier resümiert werden: Verschiedene Studien und Diskurse stellen die These auf, dass Adoptiveltern ab ihrem Eintritt als adoptionswilliges Paar ins behördliche Verfahren von vielen Seiten zugeschrieben wird, dass ihre soziale Elternschaft nicht nur nachweislich und ausdrücklich gewünscht, sondern auch gegenüber überprüfenden Instanzen erkämpft und dadurch erworben worden sei. Bereits durch die Deskription der bestehenden Verfahren kann sicher gesagt werden, dass die Adoptiveltern nach der rechtlich erfolgten Adoption eines Kindes im Unterschied zu biologischen Eltern vorab und gemäß staatlich bestimmter Standards für fähig beurteilt worden sind. Von ihnen wird folglich - im Unterschied zur biologisch gewordenen Elternschaft - eine Formalisierung und Theoretisierung normativer oder tabuisierter, privater Themen ex ante gefordert. Allerdings gehört genau die Frage danach, weshalb Menschen oder Paare Eltern oder nicht Eltern werden möchten, schon seit Längerem zu hochkomplexen, kontroversen und wohl auch divers tabuisierten westlichen Gesellschaften (vgl. z. B. Gloger-Tippelt et al. 1993, S. 21 ff.; Glass et al. 2016).

Die Idee hergestellter „Elternheit“ (in Anlehnung an Fangmeyer und Mierendorff 2017) beginnt folglich mit dem Eintritt in das amtliche Eignungsverfahren quasi als Manifestationsphase einer Entscheidung, die in unzähligen Reflexionen mit

\footnotetext{
${ }^{1}$ Zitat einer im Rahmen der Zürcher Adoptionsforschung interviewten Mutter.
} 
Partner/in, Verwandten, Bekannten und/oder Fachpersonen gefunden werden musste (vgl. Gabriel und Keller 2013, 2018; Suter et al. 2014, S. 75f.).

Die Zürcher Adoptionsstudie (Gabriel und Keller 2013, 2018), auf welcher der vorliegende Beitrag basiert, setzte deshalb bei einem Zeitpunkt ein, an dem das Verfahren in der Schweiz eröffnet wird. Verschiedene Beteiligte beginnen aus unterschiedlichen Perspektiven a-priori eine Gewährleistung des Kindeswohls zu definieren und zu überprüfen sowie anschließend auch zu gewährleisten.

In diesem Beitrag wird eine analytisch getroffene Auswahl von vorliegenden empirischen Erkenntnissen aus dieser Studie im Hinblick auf die Erfahrungen mit sozial erworbener Elternschaft im Feld der Adoption diskutiert werden. Ein besonderes Interesse gilt dabei den Fragen nach Handlungs- und Seinssicherheit sozialer Eltern in diesem normativ wie auch fachlich dichten Umfeld (vgl. Fragestellungen in Abschn. 1). Bevor auf diese Fragen anhand des empirischen Materials eingegangen wird, wird kurz das Design und die erkenntnisleitenden Fragen sowie die Methodik der Auswertung der gesamten Zürcher Adoptionsstudie vorgestellt.

\section{Die Zürcher Adoptionsstudie: Design, Fragestellung und Methodik der Auswertung}

Die ,Zürcher Adoptionsstudie“ ist eine Langzeitstudie des Instituts für Kindheit, Jugend und Familie der Zürcher Hochschule für Angewandte Wissenschaften (ZHAW), Departement Soziale Arbeit. Sie wurde von der Kantonalen Zentralbehörde Adoption des Amtes für Jugend und Berufsberatung Zürich (AJB) im Jahre 2009 in Auftrag gegeben. Die Langzeitstudie fragt für das Aufwachsen des Kindes und das System Familie nach Schutz- und Risikofaktoren in Adoptionsverläufen und wie diese längerfristig miteinander interagieren. Es geht dabei auch um die Einflüsse des Verfahrens und weiterer Kontakte mit Fachstellen und -personen auf das Zusammenleben der „,amtlich bewilligten Familien“ bzw. der ,erworbenen Elternschaft“ (Gassmann 2018).

Im Jahr 2009 wurden zuerst alle 195 Familien, die zwischen 2003 und 2009 im Kanton ein Kind zur Adoption bei sich aufnahmen, durch das Forschungsteam mittels standardisiertem Fragebogen über Verhalten und Wohlergehen des Kindes (CBCL C/YSR (C), vgl. Achenbach 1991) befragt. Die Auswertung der standardisierten Fragebögen erlaubt einen Vergleich mit einer zu erwartenden Häufigkeit, die das Instrument anhand von großen Normgruppen berechnet hat (der Fragebogen ist repräsentativ abgesichert mit Normdaten von einer Vielzahl von empirischen Befragungen weltweit und Intensitäten von Verhaltensausprägungen in verschiedenen Bereichen). Die Auswertung der teilstandardisierten, d.h. offeneren Fragen ermöglichte zudem das Sammeln von zentralen Themen, die die Eltern und deren Kinder beschäftigen. Trotz aufwändiger Standardisierung und Operationalisierung betont das CBCL-Instrument aber auch, dass es sich nicht als Diagnoseinstrument versteht, sondern nur die jeweils erhobene Perspektive (Eltern/Kinder) auf beobachtbares Verhalten erfassen kann (vgl. Achenbach et al. 2002). Deshalb empfiehlt es weitere Zugänge, wie z. B. Interviews, ergänzend beizuziehen, um ein ganzheitlicheres Bild zu erhalten. 
Aus den 119 teilnehmenden Familien im Jahre 2009 wurden im Jahr 201023 Familien bzw. Eltern ausgewählt, die in narrativ geführten Interviews (vgl. Schütze 1983) befragt wurden. Im Fokus der Auswertung der so erhobenen Erzählungen nach der Grounded Theory (vgl. Strauss und Corbin 1990; Strübing 2004; Glaser und Holton 2004) standen deren intersubjektiven Erfahrungen und Eindrücke auf dem Weg hin zur Adoptivfamilie. 2014 wurde die briefliche Befragung mit denselben Familien wie 2009 wiederholt, 2015 und 2016 folgten erneute Interviews mit den im Jahr 2010 ausgewählten Familien.

Die Studie beinhaltete in beiden bisherigen Erhebungswellen also zwei Zugänge: einen brieflichen an eine Grundgesamtheit der Familien (quantitativ und standardisiert) und einen offen-erfragenden bei ausgewählten Familien (qualitativ). Es handelt sich deshalb um ein Mixed Methods Design (vgl. Creswell et al. 2017), in dem zuerst die quantitative der qualitativen Datenerhebung vorangestellt wird und diese beeinfluss. Weil es sich um eine Längsschnittstudie handelt, beeinflusst die qualitative Erhebung zum Zeitpunkt (t1) dann auch wieder die zweite quantitative (t2) zumindest hinsichtlich der teilstandardisierten Fragen:

In beiden bisher durchgeführten, quantitativen Erhebungen (2009 (t1) und 2014 (t2)) wurden die Daten somit durch folgende Befragungsinstrumente genutzt:

- Standardisierter Fragebogen „Child Behavior Checklist“ (CBCL@) mit ca. 120 Fragen und 8 Auswertungsskalen zu Erhebung kindlichen Verhaltens und Wohls durch Befragung der Eltern;

- Standardisierter Fragebogen „Youth Self Report“ (YSR (C) mit ca. 120 Fragen und 8 Auswertungsskalen für die mit der CBCL(C) vergleichbaren Befragung von Jugendlichen zwischen 11 und 18 Jahren (an die Kinder, die jeweils bereits in dem Alter waren)

- Ein offener Zeichnungsauftrag (,Das bin ich, wenn ich mal groß bin“) für Kinder zwischen ca. 7 und 11 Jahren, da für die Kinder unter 11 kein entsprechend kompatibles Instrument versandt wurde.

Die im Folgejahr interviewten 23 Familien wurden im Sinne des Mixed Methods Design (vgl. Creswell et al. 2017) basierend auf den quantitativen Auswertungen der brieflichen Fragebögen kontrolliert so gewählt, dass sie im gesamten Sample ganz unterschiedliche, teils kontrastive Ausgangslagen repräsentierten. Eine kontrollierte Auswahl bedeutet, dass bekannte Faktoren wie zum Beispiel Bildungsniveau der Eltern, Herkunft des Kindes oder Alter des Kindes beim Eintritt im kleineren, qualitativen Sample vergleichbar verteilt und vertreten sind wie im größeren, quantitativen Sample (vgl. Bréchon 2015; Creswell et al. 2017). Bei der Auswahl für die qualitativen Interviews wurde zudem ein höheres Eintrittsalter der Kinder (4 Jahre und älter) als bekannter Risikofaktor sowie aus dem quantitativen Modul bekannte Motive und Erfahrungen der Eltern berücksichtigt. Durch die Faktorenrepräsentanz im Sample der qualitativen Erhebung kann gewährleistet werden, dass die interviewten Adoptiveltern für die Grundgesamtheit der quantitativ befragten Adoptiveltern $(N=119)$ stehen.

Die zentrale qualitative Erhebungsmethode waren narrative Interviews (vgl. Schütze 1983; Rosenthal und Loch 2002). Der Gesprächsleitfaden orientierte sich nicht an der tatsächlichen Chronologie der Ereignisse, sondern an feststellbaren 
Veränderungen in der Familienform, wie bspw. der Ankunft des Kindes. Bei den narrativen Interviews mit denselben Familien in den Jahren 2010 (t1) und 2015/16 (t2) war ein großes Anliegen, den Befragten inhaltlich und zeitlich so viel Raum wie möglich zu lassen, um an ihre Erfahrungsqualitäten zu gelangen (vgl. Schütze 1983, S. 285 f.). Ziel war es, die subjektiven Erfahrungen der Eltern zu erheben und mit diesem Fokus eine abstrakte Theoretisierung über Adoption und Elternschaft durch die Eltern zu vermeiden (vgl. Hermanns 1991, S. 185). Dies war insbesondere relevant, da die Eltern durch die Eignungsabklärungen im Rahmen des Adoptionsverfahren in theoretische Auseinandersetzungen mit Kindheit, Elternschaft und Adoption gezwungen wurden. Die Interviewfragen wurden alle offen narrativ gestellt, um den Befragten genügend Raum zu lassen für die Wahl und Betonung subjektiver Bedeutung (vgl. Schütze 1983).

Es war des Weiteren entscheidend, dass sich der Frageverlauf deutlich von der amtlichen Eignungsabklärung abgrenzen konnte, um keine (theoretisch) vorgefertigten Bilder und Geschichten zu reproduzieren. Wichtig waren hierfür insbesondere die Einstiegsfrage, die nicht chronologisch beim Kinderwunsch beginnt, sowie das jeweilige Nachfragen nach konkreten Erlebnissen und Erfahrungen.

Ziel der qualitativen Analyse, basierend auf der Grounded Theory (vgl. Strauss und Corbin 1990; Glaser und Holton 2004; Strübing 2004), war es u.a., intersubjektive Erfahrungen zur Genese von Handlungs- und Seinssicherheit sowie von Handlungs- und Seinsunsicherheit bei der adoptionsbedingten und somit nicht-biologischen Entstehung von Elternschaft auszuarbeiten.

Hierfür wurden die in den Erzählungen verborgenen Bedeutungszusammenhänge anhand der wortwörtlich transkribierten Interviews in Analyseteams entlang des offenen, axialen und selektiven Codierverfahrens (vgl. Strauss und Corbin 1990, S. 96 ff.; Glaser und Holton 2004, S. 13 f.), das auch hermeneutische Erkenntniszugänge beinhaltet, zu Schlüsselkategorien verdichtet. Diese Auswertungsmethode ermöglichte es, aus den Gesprächen mit den 23 Adoptivelternpaaren induktiv Themen und Erfahrungen im Adoptionsprozess zu rekonstruieren und zu generieren, die sich in den bisherigen Familiengeschichten übergreifend als bedeutsam erwiesen haben. Ein Vergleich zur biologischen Elternschaft ist explizit nicht im Forschungsdesign berücksichtigt. Im Zentrum stehen primär spezifische Faktoren und Kontexte der Adoption (Eberle 2014), aus welchen sich nach der Analyse theoretische Konzepte mit noch nicht absehbarer Reichweite ableiten lassen (vgl. Glaser und Holton 2004, S. 9f.). Folglich waren entsprechende Narrative der Adoptiveltern von besonderem analytischen Interesse, die zeigen, wie diese ihre Familiennormalitäten konstruierten sowie ein- und abgrenzten.

Diese Analysen ergaben eine intersubjektiv geteilte Dichotomie zwischen als ,,positiv und negativ bewerteten Einflüssen“, die subjektive Erfahrungen, Entscheidungen und Handlungen maßgeblich beeinflusst. Dabei ließen sich oft implizite oder explizite Bezugnahmen auf Ergebnisse epidemiologischer Studien zu Adoptionen (exempl. Hjern et al. 2002) feststellen:

- möglichst jung (wenige Erinnerungen bzw. Bindungsaufbau) vs. älter (viele Erinnerungen und Partizipationsmöglichkeit am Prozess) 


\begin{tabular}{|c|c|c|c|c|}
\hline $2003-2009$ & $2009(\mathrm{t} 1)$ & $2010(\mathrm{t} 1)$ & 2014 (t2) & $2015 / 16(t 2)$ \\
\hline $\begin{array}{l}\text { Paare/Fami- } \\
\text { lien adoptieren } \\
\text { Kind im Kt. ZH }\end{array}$ & $\begin{array}{l}\text { 1. Quanti- } \\
\text { tative Erhe- } \\
\text { bung }\end{array}$ & $\begin{array}{l}\text { 1. Qualitative } \\
\text { Erhebung }\end{array}$ & $\begin{array}{l}\text { 2. Quanti- } \\
\text { tative Erhe- } \\
\text { bung }\end{array}$ & $\begin{array}{l}\text { 2. Qualitative } \\
\text { Erhebung }\end{array}$ \\
\hline & $\begin{array}{l}\text { Fragebögen } \\
\text { CBCL, YSR }\end{array}$ & Interviews & $\begin{array}{l}\text { Fragebögen } \\
\text { CBCL, YSR }\end{array}$ & Interviews \\
\hline $\begin{array}{l}N=195 / 241 \\
(100 \%)\end{array}$ & $\begin{array}{l}n=119 / 130 \\
(61 \%)\end{array}$ & $\begin{array}{l}\mathrm{n}=23 \\
\text { (kontrolliert) }\end{array}$ & $\begin{array}{l}n=88 / 108 \\
(74 \% t 1)\end{array}$ & $\begin{array}{l}n=22 \\
(-1 \text { zu t1) }\end{array}$ \\
\hline
\end{tabular}

Abb. 1 Design und Sample der Zürcher Adoptionsstudie nach Erhebungswellen 1 (t1) und 2 (t2), Erhebungsmethoden (Quantitative und Qualitativ) und Sample. Die Grundgesamtheit bestand aus allen Familien $(n=195)$, die im Kanton Zürich zwischen 2003 und 2009, insgesamt 241, ein Kind adoptiert haben

- gesund (keine Zusatzherausforderungen) vs. bedürftig (kein Kinderhandel; Einschränkungen: Fortschritte möglich)

- nicht als adoptiertes Kind auffallend (sich normal fühlen können) vs. als adoptiertes Kind auffallend (offensichtliches Thematisieren der Adoption)

- Kontakt zu anderen Adoptivfamilien (Austauschmöglichkeit für Eltern \& Kinder) vs. kein Kontakt zu anderen Adoptivfamilien (Adoption nicht überbetonen)

- möglichst gute Anpassungsfähigkeit der Adoptiveltern (Eingeständnisse an das Machbare) vs. möglichst gute Anpassungsfähigkeit des Kindes (Festhalten an einem Ideal)

- Kinderwunsch (Adoption als Transformation des Kinderwunsches) vs. Adoptionswunsch (Adoption als eigenständiger Wunsch)

Die elterlichen Positionierungen innerhalb dieser Spannungsfelder rahmen ihre Erfahrungsqualitäten und tatsächlichen Handlungen und wurden in der hermeneutischen Analyse berücksichtigt (vgl. Kuckartz 2018). In Anlehnung an Kelle und Kluge (1999) war es Ziel in der Interviewanalyse - insbesondere im Schritt des selektiven Codierens (vgl. Glaser und Holton 2004, S. 13f.) - die kollektiven Sinnzusammenhänge zu verdichten, um komplexe Realitäten nachvollziehbar und systematisch zu erfassen. Die Interviews wurden hier anhand der in der empirischen Analyse gefundenen Vergleichsdimensionen (vgl. Strauss und Corbin 1990) verdichtet, in ihren Ausprägungen gruppiert und auf empirische Regelmäßigkeiten untersucht (Kluge 2000, S. 4). Die Ergebnisse der Analysen sind nachfolgend mit ausgewählten Zitaten im Text exemplarisch wiedergegeben. Sie stehen dabei nicht für sich oder eine Fallrekonstruktion, sondern für eine größere Grundgesamtheit von Erfahrungen und Bewertungen sozialer Elternschaft im Kontext Adoption.

Obschon im vorliegenden Beitrag Erkenntnisse aus den zwei qualitativen Interview-Erhebungen ( $\mathrm{t} 1$ und $\mathrm{t} 2$ ) im Zentrum stehen werden, geben auch die quanti- 
tativen Ergebnisse bereits interessante Hinweise auf die gestellten Fragen nach Erfahrung und Bedeutung von sozialer Elternschaft im Rahmen des Adoptionsprozesses. Gerade die Ausgestaltungen der Übergänge im durch die kantonalen Behörden begleiteten Eignungsverfahren, das in den ersten Erhebungszeitpunkten (t1, siehe Abb. 1) im Fokus stand und dem im nachfolgenden Abschnitt die Aufmerksamkeit gilt, machen dies deutlich.

\section{4 Übergang vom Paar zur staatlich bewilligten Elternschaft}

Teilweise sind es sehr lange Prozesse von der Interessensbekundung eines Paares, ein Kind adoptieren zu wollen, bis zur Ankunft des Kindes; nochmals dann, nach dessen Ankunft, etwa ein Jahr bis zur rechtlich gültigen Adoption. Doch erst mit der Ankunft des Kindes kann die theoretisch geprüfte, soziale Elternschaft durch das Verhalten des Kindes und des Umfeldes mehrfach und vor allem konkret auf die Probe gestellt werden.

Umso erstaunlicher waren die CBCL-Auswertungen aus der ersten quantitativen Vollerhebung (t1/2009), in welchen kaum signifikante Abweichungen in Bezug auf beobachtbares kindliches Verhalten und deren analytische Skalierung zu den zu erwartenden Werten aus der Normpopulation feststellbar waren. Falls es zu Abweichungen kam, betraf dies die überdurchschnittliche bzw. die anomal große Anzahl komplett abwesender Auffälligkeiten in einzelnen CBCL-Verhaltensskalen der Kinder. ${ }^{2}$ Dies kann als weiterer empirischer Beleg für die Existenz der in der Adoptionsforschung bekannten „Honeymoon-Phase“33 (vgl. Pinderhughes und Rosenberg 1990; Wind et al. 2007) gesehen werden. Diese Metapher umschreibt eine sozial bedingte Anfangsphase nach Übergängen bspw. zur Adoptiv-Familie, die geprägt ist von Vorfreude, von einer gewissen Furcht vor Problematisierung einzelner Situationen und Geschehnissen und/oder von einer Tendenz zur Idealisierung einer endlich erreichten Familienexistenz.

Diese bekannten Annahmen ließen sich in den Analysen der ersten narrativen Interviews (t1/2010) mit Adoptiveltern $(n=23)$ noch weiter belegen und ausdifferenzieren. So ließen sich im Rahmen der Analysen sechs Umgangsmuster entwickeln, die die Übergänge vom Paar mit Kinderwunsch zur Adoptivfamilie vor und nach Ankunft des Kindes geprägt hatten. Diese Muster der (sozial gewordenen) Eltern zur Bewältigung der herausfordernden (Um-)Gestaltungen widerspiegeln gleichzeitig erziehungswissenschaftlich relevante Einblicke, wie und wo in Übergängen zur Familie ohne Rückgriff auf eine biologische Dimension dieser Lebensform Sinn zugeschrieben wird; entsprechend lässt sich auch ablesen, wie und worin das Sinnstiftende hinterfragt werden kann. In beiden Fällen sind die folgenden sechs Er-

\footnotetext{
2 Der eindeutigste negativ abweichende Trend, der sichtbar wurde, waren auffällig viele Wahrnehmungen von Aufmerksamkeitsproblemen bei den Kindern bei 5- bis 18-Jährigen. Das Zeigen von Aufmerksamkeitsproblemen ist ein externalisierendes Verhalten.

3 Die sogenannte Honeymoon-Phase wird ursprünglich in Anlehnung an Cultural Studies verwendet, es bezeichnet das soziale Phänomen eines Zeitabschnittes indem die Unterschiede zwischen der alten und neuen sozialen Situation als überwiegend positiv wahrgenommen werden und Dissonanzen oder Enttäuschungen nicht wahrgenommen werden oder nicht thematisch werden (vgl. Oberg 2006).
} 
klärungsmuster für unterschiedliche Momente der Irritation und Seinsunsicherheit für die befragten, sozialen Eltern (und in der Konsequenz für deren Kinder) von weitreichender Bedeutung: Harmonie, Erklärung, Irritation, Verstehen, Optimismus und Gelassenheit.

\section{Harmonie: „Alles ist sehr perfekt! “4}

Die Betonung, dass nun alles perfekt sei, widerspiegelt einen positiv besetzten Wunsch nach einem Kind, einer Familie und (schließlich) einer Adoption. Mit der Ankunft des Kindes in der Familie wird dieser Wunsch nach oft jahrelangem Hoffen und Warten wahr. Ist die Betonung des perfekten Zustands dann mehr als nur Ausdruck des elterlichen Glückszustandes, sondern wird zu einer dominanten Umgangsform in alltäglichen Wahrnehmungen und Handlungen, ist oft ein harmonisierendes Muster zu erkennen. Man fürchtet sich davor, auch kleinste mögliche Abweichungen, Probleme oder einen eigenen Respekt zu sehen und zu artikulieren, und betont stattdessen die Perfektion.

2. Erklärung: „Alles wegen Adoption“

Falls das Kind die Adoptiveltern mit seinem Verhalten - meist im negativen Sinne - überrascht, liegt diese subsumptionslogische Erklärung über die nicht gemeinsame Vergangenheit und die nicht gemeinsamen Gene der Adoption sehr nahe. Insbesondere zu Beginn, nachdem man meist über Jahre hinweg mit allen möglichen Risiken einer Adoption konfrontiert worden war, scheint es sehr schwierig, überhaupt andere Verstehensmuster anwenden zu können. Allerdings sind diese Erklärungen sehr geschlossen und können im Einzelfall kaum infrage gestellt werden.

3. Irritation: „Tun andere auch so? Reagier ich richtig?“

Wenn diese Adoptiveltern sich fortwährend unsicher fühlen und zur Behebung entweder keine Wissensquelle zur Verfügung steht oder nicht genutzt werden möchte, beginnen sie sich nach Referenzen umzuschauen. Das Umschauen ist geprägt von einer starken Unsicherheit und einem (noch) fehlenden Selbstvertrauen als (Adoptiv-)Eltern. Hinzu kommt die offene Frage, wo sich überhaupt ein angemessener Vergleich finden lässt: bei biologischen Familien, bei Adoptivfamilien, bei befreundeten Familien, in Büchern oder in gesellschaftlichen Bildern?

4. Verstehen: „Woran liegt das? Weshalb diese Reaktion?“

Ein ganz anderer Umgang als das Hervorheben der Harmonie, das Bereithalten von Antworten oder der Vergleich mit anderen Normalitäten ist der Versuch einer verstehend-offenen Haltung gegenüber sich und dem Kinde. Dieses eigenständige Suchen nach möglichen Zusammenhängen in der Vergangenheit, in der Gegenwart und im Kontext ermöglicht erst eine Lernbereitschaft. Zwar werden auch unterschiedliche Referenzen beigezogen, aber die Reflexionsbereitschaft, welche dieser Umgangsform zugrunde liegt, ermöglicht Antworten ohne abschließenden Charakter. Dadurch ist der Druck kleiner und die Flexibilität in Situationen zu reagieren grösser.

\footnotetext{
4 Bei diesen Betitelungen handelt es sich nicht um wortwörtliche Zitate, sondern um sinnhafte Paraphrasierungen.
} 


\section{Optimismus: „Die Fortschritte sind unglaublich“}

Im Gegensatz zur Umgangsform „Alles ist perfekt“ fokussiert diese das Kind weniger als Bestandteil eines perfekten Gesamtzustandes, sondern sie nimmt sich deren eigenständigen Fortschritte an. Dies kann auch innerhalb einer sonst als belastend wahrgenommenen Zeit geschehen oder innerhalb eins sehr spezifischen Rahmens. Im Unterschied zur latenten Sinnebene bei „Alles wegen Adoption“ stehen hier auch nicht die Defizite, sondern die Überwindung der Defizite oder die Förderung von Ressourcen im Zentrum - spontan oder als absichtliche Strategie. Wie beim vorangehenden fragenden bzw. verstehenden Umgang scheint auch dieser einer zu sein, der selten ab Ankunft des Kindes vorkommt.

6. Gelassenheit: „Wie bei anderen auch. Jedes Kind ist anders“"

Ein deutliches Anzeichen einer weniger abhängigen, subjektiven Wahrnehmung des Kindes und auch von sich selbst als (Adoptiv-)Eltern ist diese Umgangsform, die sich loslöst von vielen Fragen, schließenden Erklärungen oder von Vergleichen. Irritationen sind bis zu einem gewissen Grad akzeptiert und ob eine Antwort wichtig ist, wird in der Situation abgeschätzt. Da die meisten Paare nach Abschluss des Verfahrens häufiger in die (unbekannte) Vergangenheit blicken, findet sich auch dieses Umgangsmuster oft erst mit der Zeit. Dies scheint auch wichtig, da eine solche Haltung zu Beginn tatsächliche Herausforderungen relativieren könnte.

Diese Umgangsmuster können phasenweise auftreten oder generell den Einstieg in das sozial bedingte Familienleben bestimmen. Jedes dieser Muster kann die Eltern bei außergewöhnlichen Ereignissen unterschiedlich stark beunruhigen und gibt dem Adoptivkind jeweils unterschiedliche Möglichkeiten der Einflussnahme. Die einzelnen Muster können sich auch ablösen, z. B., dass nach einer ersten Phase der Harmonie plötzlich jedes Verhalten des Kindes auf dessen Adoption zurückgeführt und problematisiert wird (Erklärung).

Vor allem zeigen diese Ergebnisse aus den qualitativen Interviewanalysen, dass viele Adoptiveltern latent oder explizit nach einer imaginierten Familiennormalität suchen und damit Gefahr laufen, diese im Sozialen validieren wollen zu lassen. Dies kann sie jedoch stets erneut verunsichern, anstatt dass sie sich auf die eigene Urteilsfähigkeit verlassen könnten (,Tun andere auch so?“). Die Wertungen des sozialen Umfelds üben folglich einen großen Einfluss auf deren Selbstverständnis als soziale (Adoptiv-)Eltern aus, was zur Harmonisierung (,Alles ist sehr perfekt") oder aber zur Dramatisierung (,Alles wegen Adoption“) führen kann. Besonders ungünstig wirkt es sich aus, wenn fachliche Abklärungen und Begleitungen als kontrollierend statt als unterstützend wahrgenommen werden. Dann fällt es bspw. schwerer, sich Unsicherheiten einzugestehen und Hilfe zu beanspruchen (,Alles ist sehr perfekt ${ }^{\star}$ ). Wenn hingegen Ängste, Mythen und Ideale reflektiert werden können und das Elternpaar auch konkret zu Veränderungen bereit ist (,Woran liegt das?“"), steigen die Chancen für einen gelingenden und auch selbstbewussten Start. Es scheint hier beinahe redundant, wenn abschließend zu diesen eruierten Umgangsmustern darauf verwiesen wird, dass dem Sozialen bei sozialer Elternschaft oft ein sehr großer Einflussraum auf die Ausbildung ihres Selbstverständnisses zugestanden wird. 


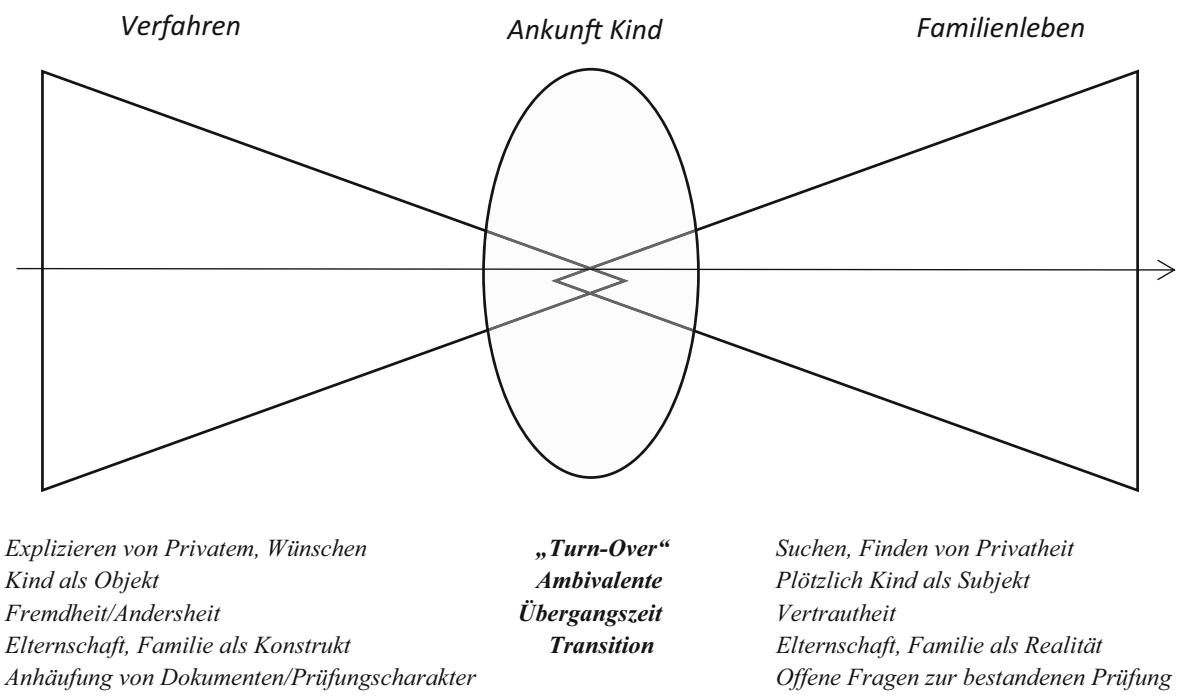

Abb. 2 „Trichtermetapher“: Visualisierung der vereinzelten Rückkehr zu Themen des „Turn-Overs“ zwischen den zwei thematischen Trichtern, die den Übergang zur Adoptionsfamilie charakterisieren

Die anspruchsvollste Phase beginnt somit mit dem positiven Bescheid sprich konkreten Vorschlag eines Kindes zur Adoption. Bei der Ankunft des Kindes steht die staatlich begleitete Eignungsabklärung zwar beinahe am Ende, das soziale Familienleben jedoch erst ganz am Anfang. Den Prozessen dieser Phase ist gemäß Zürcher Adoptionsstudie deshalb ein ausgeprägter Charakter immanent, der mit der Metapher eines „Turn-Over“ umschrieben werden kann. Nach meist jahrelangem Warten, umfassenden Überprüfungen und theoretischer Vorbereitung beginnt innert weniger Tage oder Wochen plötzlich der Familienalltag. Diese Veränderung fordert die werdenden Adoptiveltern stark - manche überfordert sie gar oder löst Ängste aus, trotz offiziell anerkannter Eignung zur Elternschaft zu versagen. Einige fühlen sich $\mathrm{zu}$ wenig gut vorbereitet, um sich konkret und vor allem auch spontan mit dem Kind auseinanderzusetzen, ihm eine mitgestaltende Rolle zuzugestehen und so gemeinsame Privatheit aufzubauen. Dieses Nadelöhr bzw. dieser Trichter zwischen zwei Bedeutungsdimensionen wird in Abb. 2 entlang der Themen, die es in den ersten Monaten und Jahren gemeinsam zu transformieren gilt, visualisiert. Sie macht deutlich, wie die Themen aus dem formalisierten Verfahren ab Ankunft des Kindes die Rollen-(Findung) der sozialen Eltern insbesondere in den ersten Jahren bestimmen. So verändert sich die im Verfahren theoretisch erschaffene „Elternschaft als Konstrukt“" in eine „Elternschaft als Realität“, das Kind als Objekt der Wünsche, des Nachdenkens und Entscheidens der Eltern wird zum „Kind als Subjekt“ mit eigenen Bedürfnissen, Geschichten und Ideen.

Die empirisch belegte, für soziale Eltern und Kinder anspruchsvolle „Turn-Over“Charakteristik im Übergang zur Familie stellt für die zweiten quantitativen und qualitativen Erhebungswellen (t2), die 2014 und 2015 durchgeführt wurden, Fragen an die weitere Entwicklung in Bezug auf das Verhalten der Kinder und dessen 
subjektive Einordnung durch die Adoptiveltern auf ihrem gemeinsamen Weg zur sozialen Elternschaft und Familie.

\section{Normalität und weitere Krisen nach den ersten Jahren als ,sozial konstruierte" Familie}

Fünf Jahre nach der ersten Befragung kann als Erkenntnis aus der zweiten quantitativen Befragung ( $\mathrm{t} 2$ 2014) festgehalten werden, dass die sogenannte „HoneymoonPhase" vorüber zu sein scheint. Dies zeigen deutliche Abnahmen im (gemäß Normalverteilung des Instruments CBCL(C) ,überdurchschnittlichen Bereich“ hin zu erwartbaren Häufigkeiten von Verhaltensauffälligkeiten bei den Kindern und Jugendlichen. Es kann somit im standardisierten und quantitativen Sinne klar von einer „Normalisierung“ gesprochen werden, die zwischen t1 (2009) und t2 (2014) in den Familien stattgefunden hat - vom Überdurchschnittlichen zum Durchschnittlichen. Im Schatten dieser Annäherung an zu erwartendes Verhalten haben sich jedoch kritische Fälle von Verhaltensproblemen, die Kinder und/oder deren soziales Umfeld stark belasten können, nicht nur gehalten, sondern in einzelnen CBCL@Skalen deutlich zugenommen.

Erziehungswissenschaftlich besonders interessant erscheint, dass Kindergarten und Schule in den offenen Antwortkategorien der zweiten quantitativen Umfrage als eine neue, einschneidende Quelle von Sorgen für die Adoptiveltern und deren Kinder auftauchen. Dazu passen auch die quantitativen Auswertungen, wonach nun die Eltern deutlich mehr aufgrund des möglicherweise sozial auffälligen Verhaltens und nicht mehr wegen des Mangels an Selbstvertrauen ihres adoptierten Kindes besorgt sind. Das bedeutet, dass nun - wohl auch im Zusammenhang mit den Übergängen in Kindergärten und Schulen - die Sorgen um Auffälligkeiten im Sozialen für die (sozialen) Eltern bedeutsamer sind als Sorgen um das Innenleben, um psychische Entwicklung und Wohlergehen des Kindes. Das Soziale im Sinne eines kollektiven Bedeutungshorizonts eines Generalized Other (Mead 1965 [1934], S. 154), der sozialen Anerkennung entziehen oder verteilen kann, kann gemäß den quantitativen Ergebnissen soziale Elternschaft ganz besonders infrage stellen oder aber umgekehrt ebendiese bestätigen. Trifft Letzteres zu, erfreuen sich die Eltern gemäß offenen Antwortkategorien des Fragebogens ab der Offenheit der Kinder, ab ihrem sozialen, hilfsbereiten Auftreten oder ihrem starken Willen. In beiden Ausprägungen machen sich viele Adoptiveltern stark von sozialen (Un-)Auffälligkeiten und Bewertungen abhängig, die durch den Eintritt in das Volksschulsystem offenbar sehr deutlich zunehmen.

Zum Zeitpunkt der zweiten narrativen Interviews mit den Adoptiveltern (2015/16) befanden sich die 22 Familien, die von den ursprünglich 23 nochmals zum Interview bereit waren, in sehr unterschiedlichen Phasen. Dies hängt auch mit dem unterschiedlichen Alter, den unterschiedlichen Entwicklungsständen sowie den unterschiedlichen Lebensgeschichten der Kinder (und Eltern) zusammen. Doch auch wenn vielerorts alltägliche Themen wie Schule, Entwicklungsschritte oder Verunsicherungen in Erziehungsfragen den Alltag bestimmen, zeigt sich in den qualitativen Ergebnissen zur rekonstruierter Genese sozialer Elternschaft deutlich, was sich in 
der quantitativen Befragung mehrfach angedeutet hatte: dass viele der erneut interviewten Familien in den vergangenen 5-6 Jahren teilweise existenzielle Krisen $\mathrm{zu}$ überwinden hatten oder diese immer noch bewältigen - entweder waren einzelne Personen (Elternteil und Kind), das ganze Familiensystem oder auch eine Einzelperson (Kind oder Elternteil) davon betroffen. Krise wird hier gestützt auf das empirische Material so verstanden, dass sie sich in erfahrener Ohnmacht bzw. Deutungsunfähigkeit manifestiert, in der die Eltern und/oder Kinder für sich keine (individuell oder sozial verträgliche) Handlungsoption mehr sehen (vgl. Gabriel und Keller 2015, S. 50f.). Als explizite Konsequenz solcher wahrgenommenen Krisensituationen wurden in den Familien dann bspw. die Polizei eingeschaltet (in 3 Fällen) oder externe Stellen (Notfallpsychiatrie, Therapien, Anlaufstellen) beigezogen. Überwiegend führten diese Krisen zu vorübergehender oder in einigen Fällen auch zu anhaltender Resignation, Frustration und Erschöpfung, die im Binnenraum der Familie bearbeitet wurden oder unbenannt und unbearbeitet blieben.

Zwar gehören aus erziehungswissenschaftlicher Sicht Rückschritte und Krisen zum Aufwachsen allgemein und zum Aufwachsen in Familien im Spezifischen dazu (vgl. bspw. Neumann 1999). Doch lassen bereits die Einblicke in die rein deskriptiven Veränderungen des qualitativen Samples zwischen den zwei Befragungszeitpunkten, wonach Sorgen der Eltern und Auffälligkeiten der Kinder zugenommen haben, aufhorchen. Im Folgenden wird auch deshalb das Erkenntnisinteresse exemplarisch auf die Dimensionen sozialer Elternschaft gelegt, die Aussagen zur Bedeutung, zur Ermöglichung wie auch ,Verunmöglichung'von familialer Zugehörigkeit erlauben.

\section{6 ,Leiblichkeit“" als ein Schlüsselkonzept von Zugehörigkeit und Herkunft, Fremdheit und Vertrauen}

Die Frage der „Leiblichkeit“ taucht in den Interviews mit den Adoptiveltern vor allem dann wortwörtlich oder noch deutlicher als sinnstiftende oder -hinterfragende Kategorie auf, wenn von den Eltern anstatt Gemeinsamkeit und Zugehörigkeit Unterschiedlichkeit und Befremdung erfahren oder gedeutet und in eine Verbindung mit der adoptionsbedingten Abwesenheit von „Leiblichkeit“ des Kindes - im Sinne der nicht vorhandenen biologischen Verwandtschaft - gebracht wird. Am deutlichsten wird dies, wenn das Adoptivkind durch bewundernde oder defizitäre bis abschätzige Betrachtungsweisen auf Distanz gehalten wird oder wenn ,leibliche Kinder“ konkret oder auch (als unerfüllter Wunsch) als Vergleichsschablone beigezogen werden. In einigen Äußerungen der Adoptiveltern wird auch deutlich, dass das ,,nicht-leibliche" Adoptivkind fremde Lust eines fremden Paares aus teils fremden bis exotischen Ländern verkörpert, fremde und unbekannte Gene besitzt, die andere Fertigkeiten und Fähigkeiten als die eigenen ausmachen. Das wird offenbar beim Heranwachsen des Kindes für die Eltern deutlicher, als in den Baby- und Kleinkindjahren (zum ersten Befragungszeitpunkt t1 fünf Jahre zuvor). Je nachdem werden Normalität und Abweichungen davon entlang dieser abwesenden leiblichen Verbundenheit explizit beim Namen genannt oder nur punktuell angedeutet. 
Zur Verunsicherung, zum grundlegenden Hinterfragen der Sinnhaftigkeit der gewählten familialen Lebensform kommt es vor allem, wenn „Leiblichkeit“ und „Adoption“ als Kontraste einander gegenübergestellt werden. So betonen zum Beispiel einige der interviewten Eltern ihre damalige bewusste Entscheidung und der daraus resultierende Eintritt in das administrativ gerahmte Eignungsverfahren als Ursprung der Adoption und erwähnen als Kontrast dazu die leibliche Lust oder den leiblichen Akt der biologischen Eltern des Kindes in abschätziger Manier. Wieder andere halten das Verhalten des Kindes, sein Körper, seine Leistungen oder sein Charakter durch überhöhte Bewunderung oder überhöhte Kritik - auch sprachlich - deutlich vom eigenen Leib fern, wie in diesen zwei Zitaten, die hier deshalb exemplarisch ausgewählt wurden, weil sie gemäß den qualitativen Auswertungen ein intersubjektives Konzept von Adoptiv-Elternschaft analytisch verdichten: „Ich bin manchmal eifersüchtig auf seine Sportlichkeit“ (Adoptivmutter 3371), „, Sie sind auch wahnsinnig robust, beide Adoptivkinder " (Adoptivvater 3197). ${ }^{5}$

Entsprechende Anmerkungen von Drittpersonen können solche Setzungen zusätzlich stützen. Auffällig ist darüber hinaus, dass viele der interviewten Adoptivmütter die Differenz zwischen „leiblicher“ bzw. biologischer und sozialer Elternschaft weitaus deutlicher betonen als die Adoptivväter. Das könnte mit der ausgebliebenen leiblichen Erfahrung durch Schwangerschaft und Geburt zusammenhängen, die Vätern auch bei biologischen Kindern nicht zugänglich sind. Ebenso ist es möglich, dass vergleichbare Themen der sozialen Väter wie die Infragestellung derer Fruchtbarkeit, Männlichkeit oder einer vorgesehenen Rolle Stammhalter schwieriger zu verbalisieren sind als ausgelassene Schwangerschaft und Geburt bei den sozialen Müttern. Am nächsten liegt aber wohl die Erklärung der ungleich verteilten Verbalisierung von Herkunft in der Tatsache, dass der biologische Vater in den Erzählungen kaum je eine Rolle, wohingegen die biologische Mutter in unterschiedlichen Zusammenhängen oft vorkommt. Wohl weil sie nicht in Konkurrenz zur eigenen Rolle steht, können die sozialen Väter darüber auch besser sprechen als die sozialen Mütter.

So nennt in einem Interview der Vater die leibliche Mutter des Sohnes stets „Ma$m i$ “ (Adoptivvater 3025). Seine Frau fällt ihm daraufhin mehrmals ins Wort um die „Leiblichkeit“ als Abgrenzung zum emotionalen und sozialen richtigzustellen: „Ja, das ist für ihn schon auch wichtig, seine leibliche Mutter" (Adoptivmutter 3025). Nicht selten entsteht, analog zu diesem kurzen Interviewausschnitt, der Eindruck, dass gerade wegen der Adoption viele Adoptivmütter versuchen, die unüberwindbare Lücke zwischen abwesender und vorhandener, leiblicher Verbundenheit als Voraussetzung von bedingungsloser Zuwendung mit erhöhtem sozialen Engagement und hoher emotionaler Präsenz zu überbrücken. Dies macht diese Mütter allerdings auch verletzlicher gegenüber möglichen Zurückweisungen des Kindes (vgl. dazu auch: Michaelsen 2017; Gassmann 2018). Diese sentimentalisierenden Phänomene zur „Leiblichkeit“, die wohl auch einen zentralen Bestandteil der „Honey-MoonPhase“ (vgl. Pinderhughes und Rosenberg 1990; Wind et al. 2007) und der Beendigung dieser Phase darstellt, kann somit auch zu Zuständen der Erschöpfung oder

\footnotetext{
5 Die ursprünglich wortwörtlichen Zitate sind für diesen Beitrag vom Schweizerdeutschen Dialekt ins Hochdeutsche übersetzt worden.
} 
der erfolglosen Sinnsuche führen - und zwar sowohl bei den Eltern als auch bei den Kindern. Und gerade da kann das Fremde dann wieder ganz besonders betont und hervorgehoben werden, wie diese Beispiele aus zwei Familien exemplarisch zeigen:

- Adoptiv-Eltern, die soziale Zugehörigkeit des Kindes infrage stellen: Soziale Eltern mit diesem Wahrnehmungsmuster, das aus den qualitativen Datenanalysen generiert wurde, nehmen für sich eindeutige und kaum überwindbare Differenzen wahr zwischen ihren adoptierten Kindern und - imaginierten oder realen leiblichen Kindern. Zumeist geht es dabei um Gefühle der Wiedererkennung, des Vertrauens und der Zugehörigkeit: „Irgendwo kommt dann schon der Punkt, wo wir finden, dass dies [dieses Kind und dessen Verhalten] fremd sei. Das ist ... dann kommt eine Distanz. Ich weiß nicht, ob das bei leiblichen Kindern im gleichen Masse aufkäme. Ja, vielleicht nicht so. Aber man ist sicher schockiert und denkt, mein Gott, das bin ich nicht." (Adoptivvater 3025, Z1810-1813)

- Kinder, die soziale Zugehörigkeit zu (sozialen) Eltern infrage stellen: Ein zweites Muster der Befremdung entsteht, wenn von Seiten der Kinder die soziale Elternschaft und somit zum Beispiel die Berechtigung der Erziehungstatsache explizit infrage gestellt wird. Oft werden als Antwort darauf dann zwei Eltern- bzw. Mutterschaftsformen konstruiert, wonach sie gleichzeitig die Eltern sind, aber auch nicht: „Also, wenn wir mit dem Kind darüber sprechen und sie irgendwie sagt: ,Du bist gar nicht meine richtige Mami, du hast mir nichts zu sagen“. Dann sag ich: ,Ja, ich bin deine Adoptivmami, aber ich bin deine Mami und du hast noch eine leibliche Mami`. Das wird von Anfang an klar kommuniziert. (...) Ich kann nicht immer einordnen, woher das jetzt kommt, was Ursachen sind. Ob's einfach daher kommt, dass er ja weiß, dass wir nicht seine leiblichen Eltern sind. So: , halt deine Klappe, geh weg “. "(Adoptivmutter 3371, Z920-925)

Adoptiveltern, die sich nur schwer vom Ideal einer Familie, das auf Schwangerschaft und den entsprechenden Dimensionen der „Leiblichkeit“ basiert, loslösen können, verstehen beim Auftauchen von Konflikten und Krisen ihre adoptionsbedingte Abweichung (einer sozial und nicht biologisch gegründeten Familie) oft plötzlich als potenziell verantwortlich für Problemstellungen und Schwierigkeiten. Die idealisierende Verwobenheit von Familie und Leiblichkeit, wird in den Interviews thematisch - wie bspw. im obigen Zitat des Adoptivvaters als Befremdung im Umgang mit dem Adoptivkind (,Ich weiß nicht, ob das bei leiblichen Kindern im gleichen Masse aufkäme“) benannt. Die imaginierte Differenz zu leiblichen Kindern kann das Potential der Enttäuschung bzw. unerfüllter Erwartungen auf allen Seiten erhöhen. Wohl auch, um sich vor zu großen Verunsicherungen durch so hergestellte Abweichungen zu schützen, konstruieren viele Adoptiveltern immer wieder Analogien zur biologischen bzw. zur leiblichen Elternschaft. So zieht zum Beispiel diese Adoptivmutter zur entsprechenden Vergewisserung der Nähe zur leiblichen Mutterschaft den Kommentar einer Freundin bei, die sie nach der Aufnahme des adoptierten Kindes (im Alter von einem Jahr) besuchte: „Da hatte ich ihn auf dem Bauch, so, und sagte, ich sei ihr vorgekommen wie eine Mutter, die soeben ein frisches Baby zur Welt gebracht hätte" (Adoptivmutter 1904, Z1934-1936). Die Erwartungen an ein „frisches Baby“ kann das knapp einjährige Kind jedoch kaum erfüllen - weder in 
Bezug auf sein bisher unabhängig von der sozialen Mutter gelebtes Leben noch auf seine körperlichen und emotionalen Bedürfnisse und Fähigkeiten.

Doch die Auslöser eines von den einen wahrgenommenen Drucks, sich möglichst einer biologisch-genetischen bzw. ,leiblichen“ Kernfamilienidee anzugleichen, werden von anderen interviewten Adoptiveltern genauso als ihr Vorteil oder gar als Befreiung davon betrachtet. Diese oft bewusste Loslösung von Analogien zur Leiblichkeit, machen die nachfolgenden drei Beispiele ebenfalls wiederkehrender Muster aus den Analysen der Interviews deutlich, die mehr die soziale Lebensgemeinschaft und weniger die biologische Familienbande als identitätsstiftendes Bild manifestieren:

1. Adoption als bewusste Wahl sozialer Elternschaft: Die Eltern in diesem Muster hatten bewusst eine Adoptionsform gewählt, die allfällige Vergleiche mit „Leiblichkeit“ möglichst frühzeitig ausschließt. So macht bspw. die Adoption eines Kindes aus Indien mit einer dunklen Hautfarbe die Nicht-Leiblichkeit äußerlich sichtbar. Wenn es zudem zum Adoptionszeitpunkt bereits 4 oder 5 Jahre alt war, konnten weder die sozialen Eltern noch das soziale Umfeld (biologistische) Baby- oder Familien-Analogien aktivieren. Von diesem Ausgangspunkt aus kann sich über die Jahre eine andere, da gelassenere und unabhängigere Selbstverständlichkeit von Zugehörigkeit entwickeln, wie bei diesem Adoptivvater: „(...) unsere Kinder ändern sich (...) unsere Kinder stehen dort, wo sie stehen, das hat zum Teil Gründe, die wir kennen, zum Teil wissen wir es nicht, zum Teil liegt es vielleicht am Charakter. Sie stehen einfach dort und wir begleiten sie auf diesem Weg, sie sind einfach unsere Kinder". (Adoptivvater 3078, Z1420-1424)

2. Älteres, ,leibliches“ Kind relativiert Bedeutung der Adoption und der sozialen Elternschaft: Die Adoptiveltern, bei welchen dieses Muster dominant ist, hatten zum Zeitpunkt der Adoption bereits ein älteres, leibliches Kind und sind zugleich sicher, dass sie deshalb in einer deutlich anderen Situation wirken, als andere Adoptivfamilien; andere Eltern in der gleichen Konstellation teilten diese Wahrnehmung nicht. Durch regelmäßige Vergleiche mit ihren Erfahrungen als leibliche Eltern sehen sie beim adoptierten Kind den Faktor Adoption zwar als vorhanden, aber nicht als im Alltag bestimmend. So fühlen sie sich bspw. auf Treffen mit anderen Adoptivfamilien nicht als Adoptiveltern: „Dann muss ich sie [die anderen Adoptiveltern] immer runterholen und sagen: „Du, das ist normal, ich habe eben den Vergleich [mit leiblichem Kind]'. Und sie haben das nicht und sie machen sich wirklich viel mehr Sorgen [um ihre Adoptivkinder]“. (Adoptivmutter 3407, Z975-977). Ähnlich distanziert sich diese Mutter eines älteren, leiblichen und eines adoptierten Kindes von anderen Adoptiveltern, die immer jede Auffälligkeit beim Kinde und im Familiengefüge mit dem Fakt Adoption erklären würden: „Die, die machen sich Gedanken über Dinge, die völlig alltäglich sind. Und die schieben alles in die Schublade Adoption “ (Z982).

3. Frühe Öffnung der Adoption als Ergänzung zur und Festigung von sozialer Elternschaft: Besonders erkenntnisreich für die Fragen dieses Beitrags zur sozialen Elternschaft erscheint das spezifische Muster einer Familie, die sich in einer von Selbstzweifeln und Konflikten bestimmten Zeit nach langem Zögern schließlich professionell beraten ließ - auch wenn die Eltern das Professionelle nicht 
explizit so benennen wollen: „Das ist nicht Familientherapie, aber einfach auch uns ein bisschen begleiten lassen. “ (Adoptivvater 3001). Im Zuge dieser gemeinsam bezogenen Beratung hatte der Adoptivsohn dann zunehmend den Wunsch äußern können, Kontakt zur leiblichen Mutter zu haben (in der Schweiz). Die sozialen Eltern nahmen dies auf und ließen es mit fachlicher Begleitung schrittweise stattfinden.

Heute treffen sie sich zirka dreimal pro Jahr. In ihrer sozialen Elternrolle fühlen sie sich nicht von der leiblichen Mutter bedroht, sondern gestärkt. Denn sie machen nämlich nur wenige Gemeinsamkeiten zwischen der biologischen Mutter und ihrem adoptierten Sohn aus und können so für sich die Bedeutung des allfälligen genetischen Einflusses und des teilweise nicht vorhandenen Widererkennens stark relativieren: „Dann denke ich manchmal: ,Was nützt es jetzt, wenn sie Johann ${ }^{6}$ begleiten würde, also würde sie sich 100 Prozent in dem Kind wiederfinden? Ich glaube, nein. “" (Adoptivmutter 3001)

Hier zeigt sich auch nochmals, dass es schwierig ist, konkrete Empfehlungen in Bezug auf häufig gestellte Fragen zur Geschwisterkonstellation oder zur Öffnung der Adoptionsform zu geben. Entscheidend ist nicht, $o b$, sondern primär wie und auch wie gemeinsam diese sozial definierten Transformationen vorbereitet, durchgeführt und begleitet sind. So kann sich ein älteres, ,leibliches“ Kind einerseits positiv auf das Finden einer ungezwungenen, eigensinnigen Balance zwischen Zugehörigkeit und Herkunft auswirken, die vor Verunsicherungen schützt. Gleichzeitig verweisen biologistische Vererbungsideen, „Gene“ und ein entsprechendes Wiedererkennen der Eltern im „Leiblichen“ auch auf eine unüberwindbare - möglicherweise ebenso konstruierte - Anomalität des adoptierten Kindes, auf das Ausbleiben von gemeinsamer Herkunft und die dadurch stets zu legitimierende Frage nach Zugehörigkeit.

\section{Vulnerable (soziale) Kinder, vulnerable (soziale) Eltern, vulnerable (soziale) Familien?}

\section{1 ,Das Geheimrezept ist einfach ganz normal zu sein“6}

Überhöhte Vorstellungen einer biologischen Herkunft und Imaginationen eines (endlich) erfüllten Kinderwunschs oder das stete Suchen nach Analogien zu blutsverwandten Familien können zum Risiko beitragen, dass dem Kind, den Adoptiveltern und dem ganzen Familiensystem das Finden eines davon unabhängigen Eigensinns verwehrt bleibt. Damit Kinder gestaltend und als Subjekte sichtbar teilhaben können, müssen die (sozialen) Eltern Bereitschaft und Flexibilität zeigen, die eigenen Vorstellungen auch zu Gunsten der Vorstellungen von Kindern zurückzunehmen (vgl. Corleis und Keller 2017, S. 167). Die Ergebnisse der qualitativen Interviewanalysen im Rahmen der Zürcher Adoptionsstudie verweisen jedoch darauf, dass viele adoptierte Kinder zumeist genau dann als abweichend identifiziert werden, wenn sie bspw. versuchen durch ihr Verhalten als eigenständig wahrgenommen zu werden,

\footnotetext{
${ }^{6}$ Name anonymisiert.
} 
ihre Vorstellungen einzubringen oder auch Änderungen zu erzwingen. So kann in einem dadurch ausgelösten Konflikt oder in einer Krise der Grund immer im adoptierten, genetisch-fremden Kind gesucht werden, damit die Eltern sich und ihre Idee von Familie nicht ändern müssen - wie es dieser Adoptivvater tut mit seiner Äußerung über einen als befremdend festgestellten Wandel des adoptierten Sohnes vom braven Kleinkind, das ihm wie sein eigenes vorkam, zu einem Jungen, der ihm immer fremder wird: ,Wir haben nicht mehr das gleiche Kind“ (Adoptivvater 3414). Nachdem sein Sohn ihm viel Geld gestohlen hatte, verbindet er seine Enttäuschung mit einem gegebenen, wohl nicht veränderbaren (auffälligen) Wesen, mit dem „HaltSo-Seins“ des Kindes. „Das hat mich eigentlich enttäuscht, aber irgendwie muss ich sagen: ja, er ist jetzt halt so" (Adoptivvater 3414).

Eine explizite Vergleichsschablone zum „Normalen“, dem ihr adoptiertes Kind nicht entspreche, zieht diese dadurch zunehmend genervte Mutter bei. Sie verweist dabei auf ihr ,normales“ bzw. leibliches und 4 Jahre jüngeres Kind, das nach der Adoption zur Welt $\mathrm{kam}^{7}$ : ,Manchmal ist es gut, wenn man sieht, wie das Normale ist, andererseits ist es manchmal halt auch ... Wenn man sieht, was der 5-Jährige schon alles kann und der 9-jährige kann es immer noch nicht, dann nervt das manchmal halt auch" (Adoptivmutter 3032, Z. 199).

Im Hinblick auf die metaphorische Sprachebene noch deutlicher kritisiert dieser Vater, dass sein adoptierter Sohn sich mit seinem auffälligen Verhalten immer von außen in das „Zentrum der Familie“ manövriere und von dort aus die Familie zu dominieren versuche: „Er hat stets das Gefühl, er müsse unser Familienleben dominieren. Er müsse sich ins Zentrum rein manövrieren, egal wie" (Adoptivvater 2034, Z. 476).

Im ungünstigsten Falle schlagen von den Eltern so gedeutete Situationen, die darauf angewiesen wären, dass sich die Familienmitglieder vertrauen und umeinander besorgt sind, in als existenziell wahrgenommene Bedrohungen um. So glaubten diese Eltern, nachdem ihre Tochter oft Krankheiten vorgetäuscht habe, den Symptomen einer akuten Notsituation ihres Kindes (Blinddarm) nicht. Deshalb brachten sie es viel zu spät ins Spital, was dem Kinde beinahe das Leben gekostet hätte: „Sie hatte sich wieder mal gekrümmt und so und ich habe gedacht: ,ja, ja okay ' (...). Aber genau dann war es natürlich ernst" (Adoptivmutter 1018, Z. 498).

Es mag paradox klingen, aber weil viele Eltern sich so sehr wünschen, einfach (endlich) „,nur“ Eltern, ,nur“ Familie sein zu können und sein zu dürfen, hindert sie ein hartnäckiges Aufrechterhalten ebendieses Wunsches häufig daran. Denn um dieses Ziel zu erreichen, werden immer wieder Vergleiche zu anderen gezogen, wird besonders viel Engagement gezeigt, fühlt man sich durch andere beobachtet und auch bewertet. Das führt dazu, dass das fortwährende Orientieren an sozialen Normen anstatt zu Rollen- und Handlungssicherheit eher zu teilweise ausgeprägten Rollenund Handlungsunsicherheit oder auch zu Enttäuschungen oder Resignationen führt. Dieser wahrgenommene Druck, aus möglichst jeder Situation eine besonders ,,nor-

\footnotetext{
7 Ein in der Adoptionsforschung bekanntes Phänomen, dass Mütter nach Jahren auch medizinisch unterstützten, erfolglosen Versuchen der Befruchtung teilwiese wenige Monate nach der Adoption eines Kindes schwanger werden. In unserem qualitativen und diesbezüglich nicht repräsentativ für eine Gesamtheit geltenden Sample kam dies in 3 von 23 Familien so vor.
} 
male" Familiensituation zu gestalten, ist für viele spürbar, wie das verunsichernde Suchen dieses Adoptivvaters nach einer Normalitätsbewertung ihres Familienalltags im folgenden Zitate deutlich macht: ,Wie soll ich sagen, es ist ganz normal, also, es gibt keine großen Unterschiede, also, eigentlich, also " (Adoptivvater 3197).

Falls sich die Kinder in diesen Suchbewegungen ihrer Adoptiveltern nach Normalitäten nicht erkannt oder anerkannt fühlen, zeigen sie gemäß den geführten Interviews oft einen erfahrungsbasierten (und nicht bloß diagnostizierten) Grund und eine hohe Fähigkeit, gezielt diese verletzlichen Zonen ihrer Adoptiveltern zu adressieren: „Zum Teil kennt er uns besser als wir uns selber kennen. Und das ist auch das, wo es einem etwas unheimlich wird “ (Adoptivmutter 3414). Dadurch können sie die ihnen zugesprochene Bedeutung, von der sie sich als objekthafte Träger/innen wahrnehmen, gezielt manipulieren. Falls diese teilweise auch harte oder brutale Manipulation als böswilliges Verhalten missverstanden wird, indem beispielsweise dem Kind vorgeworfen wird, ihm nie mehr ,vertrauen zu können innerhalb der Familie " (Adoptivvater 3414), dann können sich die Kinder darin bestätigt fühlen, dass die fehlende biologisch-familiale Verbindung keine bedingungslose Zugehörigkeit erlauben.

Wird dasselbe Verhalten von den Eltern hingegen entgegen der kindlichen Erwartungen als Ausganslage genommen, grundlegende ideelle und/oder strukturelle bzw. systemische Veränderungen vorzunehmen, ermöglicht dies den Kindern zu lernen, dass sie auch zur Herstellung von neuen Gemeinsamkeiten, von hoher Verbindlichkeit beitragen können, auch wenn sie sich hierfür als „Grenzgänger“ (Adoptivvater 3371) vielleicht grenzwertig verhalten mussten.

\section{2 „Wir müssen wieder Frieden finden in unserer Familie, und das klappt mit dir nicht"“}

Als entscheidende Differenz zwischen den Übergangsgeschehen vom Paar zur sozialen Elternschaft hat sich erwiesen, welche Möglichkeiten soziale Eltern und deren Kinder - durchaus auch als Resultat eines Konflikts oder einer Krise - erkennen oder auch bekommen, um sich in der sozial gegründeten Lebensgemeinschaft selbst sein und einbringen zu dürfen, anstatt normative Erwartungen Dritter zu erfüllen. In vielen Familien wurde jedoch deutlich, dass Konflikte und Krisen (falls es zu solchen kam) von den Eltern mit einem möglichen Scheitern ihres Vorhabens, Eltern zu werden und zu sein, in Verbindung gebracht wurden. Sie versuchen dann häufig nicht zu ergründen, weshalb es zur Krise kam, sondern, wer die Schuld daran trage. Zumeist ist es dann der Faktor Adoption, manchmal das Kind, selten Externe wie Schule oder Nachbarschaft und noch seltener die Eltern selbst. Entsprechend wurden auch in mehreren der interviewten Familien das adoptierte Kind - und nicht etwa die Eltern oder die ganze Familie - in verschiedene externe Hilfsangebote gebracht, die von verschiedenen „Kursen“ über „Psychologin“, „psychologische Abklärungen“ bis zur „Körpertherapie“ reichen können. Insbesondere psychologische Abklärungen stellten zumeist eine Reaktion auf Konflikte oder irritierendes Verhalten dar wie bei dieser Mutter, nachdem ihr Kind ihr gegenüber körperlich aggressiv geworden war: „,(..) und ich hatte dann einfach sofort gesagt: ich möchte das abgeklärt haben " (Adoptivmutter 3371). 
Die häufigsten Diagnosen sind „Bindungsstörung“, „frühkindliche Traumatisierung“ oder „ADHS“. Diese Diagnosen mögen korrekt sein und können mit allen (inklusive den aktuellen) Erfahrungen des Kindes zusammenhängen und wichtige Interventionen initiieren. Sie bestätigen aber zugleich auch die Adoptiveltern in ihrer Wahrnehmung, dass nicht sie, sondern das Kind Probleme habe: „Der Psychologe sieht wirklich die Problematik, die unser Adoptivsohn hat, und es ist halt einfach auch schön, wenn wir als Eltern eine Bestätigung bekommen: Wir sind nicht total ,gaga", sondern dass Kind hat wirklich große Probleme“ (Adoptivvater 2034, Z. 396f.).

So sahen auch die Eltern im folgenden Zitat den Weg zurück zu familialem Frieden und Vertrauen nur über die Androhung eines Ausschlusses des Kindes (der schließlich nicht tatsächlich stattfand, aber dennoch emotional bestehen blieb): „, Wir müssen wieder Frieden finden in unserer Familie und Vertrauen und offensichtlich klappt das mit dir nicht" (Adoptivmutter 3414). Wie in zwei weiteren Familien des qualitativen Samples wird dieses Kind in der Folge während der Woche fremdbetreut (unterschiedliche Angebote der stationären Kinder- und Jugendhilfe).

Wenn Adoptiveltern hingegen in ihrer Lösung einer Krise statt Differenzen Gemeinsamkeiten und Vertrauen schufen, konnte allen Beteiligten eine Lebensgemeinschaft angeboten werden, die bereit war, sich fortlaufend neu zu definieren und sich so den Bedarfslagen des Kindes (und weiterer Beteiligten) möglichst gut anpasste. So können Gemeinsamkeiten geschaffen und Entfremdungen vermieden werden. Ob in dieser Lebensgemeinschaft immer auch „Elternschaft“ stattfindet, musste sich immer wieder in Aushandlungen ergeben, wobei sie auch mal nicht stattfinden darf. So streichen die Eltern im nachfolgenden Zitat ein von Krisen geprägtes Jahr gänzlich aus dem Familienkalender: „Wir hatten keine Luft mehr (...) Das Jahr können wir aus der Agenda streichen “ (Adoptivvater 3078).

Dennoch zeigt sich im empirischen Material, dass bei allen Familien, auch nach einer gelungenen Bewältigung einer Krise, eine sogenannte ,Verletzbarkeit“ (vgl. Gassmann 2018) zurückbleibt, die unter den vorgefundenen Bedeutungszusammenhängen sozialer Elternschaft erschaffen wurde und sich in gewissen Momenten und Situationen plötzlich wieder manifestieren kann. Vulnerabilität ist dabei nicht zu verwechseln mit einer Krankheit, einem Trauma oder einer Opfererfahrung. Sie zeichnet sich durch eine häufig latent bestehenbleibende Verletzbarkeit in bestimmten Themenbereichen der Elternschaft aus.

Es lässt sich somit festhalten, dass die soziale Elternschaft im Rahmen von Adoptionen zweifellos herausfordernde Themen beinhaltet, die mit realen sozialen Erfahrungen und subjektiven Deutungen ebendieser in einer durch behördliche Eignungsverfahren erworbenen, intergenerativen Lebensgemeinschaft zusammenhängen. Sie hängen zusätzlich aber auch mit bewussten oder unbewussten Beweggründen zur Adoption seitens der Adoptiveltern, mit oft auch schwierigen Erfahrungen des Kindes vor der Adoption sowie mit den gemeinsamen Erfahrungen ab Zeitpunkt der Ankunft des Kindes zusammen. Diese so gewachsenen Themen können je nach Ausgestaltung zu hochsensiblen Voraussetzungen in der Ausgestaltung einer Rolle als Eltern werden. Dies geschieht offensichtlich nur sehr selten während den ersten Jahren nach der (sozialen) Familiengründung, sondern erst nach einigen Jahren, sprich: nachdem die sogenannte Honeymoon-Phase, in der sich alle besonders viel Mühe geben und Dissonanzen ausblenden, vorüber ist. Um verletzt zu werden sind 
nicht immer Impulse von außen notwendig. Manchmal beschäftigen sich Adoptiveltern oder -kinder auch selbst derart intensiv mit möglichen Zuschreibungen oder Besonderheiten ihrer Situation, dass sich zum Beispiel Adoptiveltern selbst die Legitimität ihrer Elternrolle infrage stellen. Das bedeutet, dass der Druck, alltägliche Geschehnisse permanent anhand eines imaginierten Ideals von Normalität zu bewerten, genauso Unsicherheiten und Gefühle von Kontrollverluste auslösen kann wie explizite Stigmatisierungen oder Diskriminierungen durch Dritte.

\section{Gelungene und gescheiterte Familien - Reflexionen zur normativ anfälligen Logik von Elternschaft im Fachdiskurs}

Abschließend soll die Aufmerksamkeit nochmals der übergreifenden, erziehungswissenschaftlich relevanten Frage gewidmet werden, wie unterschiedliche Benennungen und Begründungen einer legitimen Elternschaft die Handlungs- und Seinssicherheit von nicht-biologischen Eltern beeinflussen können. Der durch ein Eignungsverfahren gerahmte Übergang vom adoptionswilligen Paar zu sozialen Eltern basiert jeweils auf internationalen sowie nationalen juristisch-formellen Gesetzgebungen (vgl. Maywald 2008). Deutlich wurde, dass zwar einige formalisierbare Kriterien zur ex-ante Überprüfung ebendieser Eignung für eine soziale (rechtliche wie auch familiale) Elternschaft wie ein guter Leumund oder genügend Wohnraum noch leicht $\mathrm{zu}$ erheben, zu verbalisieren und zu explizieren sein mögen. Die meisten anderen bleiben auch für ausgebildete Fachkräfte unzugänglich und fließen dafür implizit, zwischen den Zeilen umso deutlicher in die dichotom ausgeprägten Verfahrens- und Bewertungsschritte mit ein (vgl. Keller et al. 2017).

Entsprechend haben die Ergebnisse der Zürcher Adoptionsstudie gezeigt, dass viele Paare bzw. künftigen sozialen Eltern bereits während und nach dem Eignungsverfahren eigene Referenzsysteme aufzubauen beginnen, da sie die formalisierten und strukturgeleiteten Kriterien guter Elternschaft - nach einer unterschiedlich lang dauernden Honeymoon-Phase - zunehmend hinterfragen. Zu den eigenen Referenzgrößen gehören dann bspw. Medien, Fachwelt und vor allem auch andere Adoptiveltern.

Weil Fachkräfte und Betroffene auf Anhaltspunkte angewiesen sind, um Aussagen zu (sozial-)elterlicher Verantwortung oder zur Gewährleistung des Kindeswohls zu erlangen (vgl. Thieme 2013), müssen sich auch erziehungswissenschaftliche Forschungs- und Theoriediskurse sorgfältig mit möglichen normativen Setzungen hinter ihren Begrifflichkeiten und Argumentarien auseinandersetzen: Mit der Erhebung welcher Perspektive gelangt sie zu welchen Erkenntnissen - und zu welchen nicht? Welche Begriffe sind anfällig auf Missverständnisse? Eine Folge einer nach wie vor stark quantitativ orientierten und latent defizitorientierten Adoptionsforschung ist bspw. eine verbreitete Dichotomie und Isolierung von relevanten Dimensionen positiver oder negativer Adoptionsverläufe (vgl. bspw. Kasten 2006). Vor dieser unklaren Wissenslage werden oft undifferenzierte Adoptions- und Familienmythen verallgemeinert und verbreitet (vgl. Pinderhughes et al. 2015), die einen von den beteiligten Menschen ausgehenden Aufbau von Vertrauen und Selbstvertrauen verhindern (vgl. Michaelsen 2017). Solche Prozesse scheinen im Feld der Adoption 
aus Sicht der Forschung schneller und intensiver zu greifen, als dies aus anderen erziehungswissenschaftlichen und sozialpädagogischen Forschungsfeldern bekannt ist (vgl. Gabriel und Keller 2013, 2018; DJI 2017a). Durch die formalisierte Auseinandersetzung mit der imaginierten Form anstatt mit der Art und Weise, wie soziale Elternschaft real gelebt wird (vgl. DJI 2017a), können die vielen wichtigen Prozesse und Übergänge, die erst nach der Ankunft des Kindes im Familienkontext tatsächlich anzugehen wären, zusätzlich aus dem Blick geraten. Diese Entwertung konkreter sozialer Erfahrung führt dazu, dass oft in dichotomer Logik zwischen Gelingen und Scheitern der (sozialen) Elternschaft gedacht und argumentiert wird (vgl. bspw. Keller 2018). Dies wurde im dargelegten Schlüsselkonzept der „Zugehörigkeit" besonders deutlich.

Die hier diskutierten, ausgewählten Ergebnisse der Zürcher Adoptionsstudie (Gabriel und Keller 2013, 2018) zeigten, welche Muster die Manifestation von Zugehörigkeit in sozial begründeten Familien verhindern könnten. Sie zeigen jedoch vor allem auch auf, was unabhängig von Konzepten wie „Leiblichkeit“ oder sozialen Voraussetzung von „Familie“ für alle intergenerativen Lebensgemeinschaften - ob biologisch, genetisch, psychisch, sozial und/oder familial begründet oder nicht Zugehörigkeit ermöglicht. Zugehörigkeit stellt sich in keiner Familienform automatisch ein, sondern muss fortwährend ausgehandelt, auf die Probe gestellt und so verbindlich entwickelt werden dürfen. Könnten die Beteiligten Elternschaft offen und ehrlich vor ihren idealisierenden Bildern und Normen diskutieren und entsprechend auch relativieren, könnte dies eine gute Grundlage für einen offenen Anfang von Familie schaffen. Das behördliche Eignungsverfahren, das es zu bestehen gilt, sowie gesellschaftliche wie auch wissenschaftliche Diskurse erschweren eine solch eigensinnige Sinngebung und Rollenfindung immer wieder. Scheinbar kehren sie im Erleben der Beteiligten die eigentlich offene Grundlage zur Gründung einer anderen, eigensinnigen familialen Lebensform oft in ihr Gegenteil, in mit Idealen und Ängsten bestimmte Erfahrungsbereiche. Dabei geht vergessen: Im subjektiven Erleben im Alltag ist man nicht entweder eine soziale, biologische oder eine andere typisierbare Form von Eltern oder Kind, sondern zumeist situationsbedingt sowohl als auch - oder auch weder noch.

Diese Erkenntnisse stützen die Annahme, dass explizite Fragen nach Elternschaft allgemein unbeabsichtigte und unberechenbare Konsequenzen haben können - auch weil die Differenzen zwischen „sozialer Elternschaft“ und „biologischer Elternschaft" diffuser geworden sind (vgl. Eggen 2018, S. 185). Bereits 1999 schlug Smith, zur Klärung und normativ weniger anfälligen Orientierung, vor den Terminus ,Parenting " mehr zu etablieren: „The word parenting is clearly action oriented. Parenting means assuming responsibility for the emotional, social, and physical growth and development of a child“" (Smith 1999, S. 9). Demnach müsste es in den erziehungswissenschaftlichen Diskursen weniger um Elternschaft als Recht am Kind gehen, sondern vielmehr um, elterliche Sorge und Verantwortung ' für das Kind. Der Begriff beinhaltet alles, ,was Eltern aktiv zur Förderung ihres Nachwuchses unternehmen“ (Gabriel 2001, S. 73). Wenn zudem mitbedacht wird, dass es sich gemäß Smith dabei nicht zwingend um die Aktivitäten der „biologischen Eltern“ handeln muss, so kann von einem idealtypischen Handeln zum Wohle des heranwachsenden Menschen bzw. der nächsten Generation gesprochen werden. Der Begriff ,Parenting ‘ 
wird im deutschsprachigen Raum im Unterscheid zum englischsprachigen allerdings primär im Kontext der Beratungsliteratur verwendet (vgl. Grubenmann 2009).

Trotz der genannten Vorteile eines Terminus müsste aber bei einem allfälligen Transfer in den deutschsprachigen Diskurs bedacht werden, dass er im erziehungswissenschaftlichen Sinne das ideologische Kernstück nicht automatisch zu verändern vermag, weil Dreh- und Angelpunkt die Kernfamilie bleiben könnte (Grubenmann 2009, S. 661). Falls dies aber mitbedacht würde, könnte damit vielleicht die hier vorgeschlagene, diskursive Basis gestärkt werden, die auch vielen aktuell bestehenden, hybriden Elternmodellen zu mehr Handlungs- und Seinssicherheit verhelfen könnte. In diesem Sinne würden bspw. Eignungsabklärungen oder Diagnoseverfahren soziale Eltern in unterschiedlichen Zusammenhängen weniger prüfen und bewilligen, sondern im erziehungswissenschaftlichen Sinne sich dieser auszugestaltenden Verantwortung verstehend und befähigend annähern. Der Übergang vom Paar zur Elternschaft bliebe in der Konsequenz inhaltlich und zeitlich offener und würde so - dies scheint entscheidend - den Fokus auf das Kind anstatt auf unterschiedliche Typisierungen elterlicher Zugehörigkeit ermöglichen.

Open Access Dieser Artikel wird unter der Creative Commons Namensnennung 4.0 International Lizenz veröffentlicht, welche die Nutzung, Vervielfältigung, Bearbeitung, Verbreitung und Wiedergabe in jeglichem Medium und Format erlaubt, sofern Sie den/die ursprünglichen Autor(en) und die Quelle ordnungsgemäß nennen, einen Link zur Creative Commons Lizenz beifügen und angeben, ob Änderungen vorgenommen wurden.

Die in diesem Artikel enthaltenen Bilder und sonstiges Drittmaterial unterliegen ebenfalls der genannten Creative Commons Lizenz, sofern sich aus der Abbildungslegende nichts anderes ergibt. Sofern das betreffende Material nicht unter der genannten Creative Commons Lizenz steht und die betreffende Handlung nicht nach gesetzlichen Vorschriften erlaubt ist, ist für die oben aufgeführten Weiterverwendungen des Materials die Einwilligung des jeweiligen Rechteinhabers einzuholen.

Weitere Details zur Lizenz entnehmen Sie bitte der Lizenzinformation auf http://creativecommons.org/ licenses/by/4.0/deed.de.

\section{Literatur}

Achenbach, T.M. (1991). Manual for the child behavior checklist/ 4-18 and 1991 profile. Burlington: University of Vermont Department of Psychiatry.

Achenbach, T.M., Dumenci, L., \& Rescorla, L. A. (2002). Ten-year comparisons of problems and competencies for national samples of youth: self, parent, and teacher reports. Journal Of Emotional And Behavioral Disorders, 10(4), 195-203.

Ader, S., Schrapper, C., \& Thiesmeier, M. (Hrsg.). (2001). Sozialpädagogisches Fallverstehen und sozialpädagogische Diagnostik in Forschung und Praxis. Münster: Votum.

Ballard, R. L., Goodno, N. H., Cochran, R. F., \& Milbrandt, J. A. (Hrsg.). (2015). The intercountry adoption debate: dialogues across disciplines. Newcastle upon Tyne: Cambridge Scholars Publishing.

Beck, S. (2014). Zur Herstellung von Familie im Zeitalter der transnationalen Reproduktionsmedizin. In K. Jurczyk, A. Lange \& B. Thiessen (Hrsg.), Doing Family. Warum Familienleben heute nicht mehr selbstverständlich ist (S. 144-158). Weinheim: Beltz.

Bernard, A. (2014). Kinder machen. Neue Reproduktionstechnologien und die Ordnung der Familie. Samenspender, Leihmütter, Künstliche Befruchtung. Frankfurt a. M.: S. Fischer.

Bitter, S., Bangerter, A., \& Ramsauer, N. (2019). Adoptionen von Kindern aus Sri Lanka in der Schweiz, 1973-1997. Zur Praxis der privaten Vermittlungsstellen und der Behörden. Historische Analyse betreffend das Postulat Ruiz, 17.4181 im Auftrag des Bundesamts für Justiz. Bern: unveröffentlichter Bericht zuhanden des Bundesamts für Justiz. 
Bréchon, P. (2015). Random sample, quota sample. The teachings of the EVS 2008 survey in France. Bulletin of Sociological Methodology, 126(1), 67-83.

Cinkl, S., \& Krause, H.-U. (2014). Praxishandbuch Sozialpädagogische Familiendiagnosen. Verfahren Evaluation - Anwendung im Kinderschutz (Bd. 2). Opladen: Leske + Budrich.

Corleis, T., \& Keller, S. (2017). Fremdplatziertes Wohnen - Zwischen Möglichkeiten und Widersprüchen: Kindliche Perspektiven auf Aufwachsen am anderren Ort im Vergleich. In M. Meuth (Hrsg.), WohnRäume und pädagogische Orte. Erziehungswissenschaftliche Zugänge zum Wohnen (S. 149-170). Wiesbaden: Springer VS.

Creswell, J. W., Plano, C., \& Vicki, L. (2017). Designing and conducting mixed methods research (3. Aufl.). Los Angeles: SAGE.

DJI (Hrsg.). (2017a). Dossier Adoptionen in Deutschland. Bestandsaufnahme des Expertise- und Forschungszentrums Adoption Kurzfassung. München: Eigenverlag.

DJI (Hrsg.). (2017b). Besuchskontakte nach Adoption und Formen schwacher Adoption. Rechtsvergleichende Studie unter Einbeziehung des schweizerischen, französischen, italienischen, spanischen, griechischen, englischen und US-amerikanischen Rechts. Eine Expertise für das Expertise- und Forschungszentrum Adoption (EFZA). München: Eigenverlag.

Eberle, T. (2014). Subjektive Erfahrung, intersubjektive Verständigung und Sozialität. Phänomenologische Erörterungen. In A. Proferl \& N. Schröer (Hrsg.), Wissen und Kommunikation (S. 143-161). Wiesbaden: Springer VS.

Eggen, B. (2018). Multiple Elternschaft - Zur neuen Normalität von Elternschaft. RPsych Rechtspsychologie, 4, 181-207.

Fangmeyer, A., \& Mierendorff, J. (2017). Kindheit und Erwachsenheit in sozialwissenschafticher Theoriebildung. Weinheim: Beltz Juventa.

Gabriel, T. (2001). Forschung zur Heimerziehung. Das Beispiel Grossbritannien. Weinheim: Juventa.

Gabriel, T., \& Keller, S. (2013). Die Zürcher Adoptionsstudie. Kinder und Adoptiveltern in den ersten Jahren. Zürich: ZHAW Hochschule für angewandte Wissenschaften.

Gabriel, T., \& Keller, S. (2015). Krisen und Transitionen im Lebenslauf. In A. M. Riedi, M. Zwilling, M. Meier Kressig, P. Banez Bartoletta \& D. Aebi Zindel (Hrsg.), Handbuch Sozialwesen Schweiz (S. 47-59). Bern: Haupt.

Gabriel, T., \& Keller, S. (2018). Familiennormalitäten in Übergängen nach der Adoption: ausgewählte Einblicke in die Ergebnisse der Zürcher Adoptionsstudie. Fachtagung „Leben in Adoptiv- und Pflegefamilien - Normalitäten und Krisen“. https://pa-ch.ch/wp-content/uploads/2018/11/Referat_ThomasGabriel-und-Thomas-Keller.pdf. Zugegriffen: 10. März 2020.

Gassmann, Y. (2018). Verletzbar durch Elternschaft. Balanceleistungen von Eltern mit erworbener Elternschaft - Ein Beitrag zur Sozialpädagogischen Familienforschung. Weinheim: BeltzJuventa.

Glaser, B. G., \& Holton, J. (2004). Remodeling grounded theory. Forum Qualitative Social Research, 5(2), $1-22$.

Glass, J., Simon, R.W., \& Andersson, M.A. (2016). Parenthood and happiness: effects of work-family reconciliation policies in 22 OECD countries. American Journal of Sociology, 122(3), 886-929.

Gloger-Tippelt, G., Gomille, B., \& Grimmig, R. (1993). Der Kinderwunsch aus psychologischer Sicht. Opladen: Leske + Budrich.

Grubenmann, B. (2009). Parenting. In S. Andresen, R. Casale, T. Gabriel, R. Horlacher, S. Larcher Klee \& J. Oelkers (Hrsg.), Handwörterbuch Erziehungswissenschaft (S. 652-665). Weinheim: Beltz.

Heine, H. (1827). Philister in Sonntagsröcklein. In Buch der Lieder, Lyrisches Intermezzo. Hamburg: Hoffmann und Campe.

Hermanns, H. (1991). Narratives Interview. In U. Flick, E. V. Kardorff, H. Keupp, L. V. Rosenstiel \& S. Wolff (Hrsg.), Handbuch Qualitative Sozialforschung (S. 182-185). München: Psychologie Verlags Union.

Hjern, A., Lindblad, F., \& Vinnerljung, B. (2002). Suicide, psychiatric illness, and social maladjustment in intercountry adoptees in Sweden: a cohort study. The Lancet, 360(9331), 443-448.

Jurczyk, K. (2014). Familie als Herstellungsleistung. Hintergründe und Konturen einer neuen Perspektive auf Familie. In K. Jurczyk, A. Lange \& B. Thiessen (Hrsg.), Doing Family. Warum Familienleben heute nicht mehr selbstverständlich ist (S. 50-70). Weinheim: Beltz.

Kasten, H. (2006). Scheitern von Adoptiv- und Pflegeverhältnissen. In H. Paulitz (Hrsg.), Adoption: Positionen, Impulse, Perspektiven: ein Praxishandbuch (S. 242-270). München: C.H. Beck.

Keller, S. (2018). Schwierigkeiten in Pflege- und Adoptivfamilien: wann ist es eine Krise? Netz: Zeitschrift Pflege- und Adoptivkinder Schweiz, 3, 20-23. 
Keller, S., Gabriel, T., \& Eberitzsch, S. (2017). Fallverstehen in der Fremdplatzierung: wie kann im Dialog zwischen Praxis und Theorie neues Reflexionswissen entstehen? In H. Messmer (Hrsg.), Fallwissen. Wissensgebrauch in Praxiskontexten der Sozialen Arbeit (S. 63-92). Opladen: Barbara Budrich.

Kluge, S. (2000). Empirisch begründete Typenbildung in der qualitativen Sozialforschung. Forum Qualitative Sozialforschung, 1(1), 1-11.

Kelle, U., \& Kluge, S. (1999). Vom Einzelfall zum Typus: Fallvergleich und Fallkontrastierung in der qualitativen Sozialforschung. Opladen: Leske + Budrich.

Kuckartz, U. (2018). Qualitative Inhaltsanalyse, Methoden, Praxis, Computerunterstützung. Weinheim: Beltz Juventa.

Maywald, J. (2008). Internationale Adoptionen - Stärkung oder Schwächung von Kinderrechten? FamiliePartnerschaft-Recht, 10, 499-502.

Mead, G.H. (1965). Mind, self and society. Chicago: University of Chicago Press.

Merritts, A. (2016). A review of family therapy in residential settings. Contemporary Family Therapy, $38(1), 75-85$.

Michaelsen, A. (2017). Kippbilder der Familie. Ambivalenzen und Sentimentalität moderner Adoption in Film und Video. Bielefeld: transcript.

Neumann, K. (1999). Aufwachsen in Familien. Zur Situation der Kinder aus pädagogischer Perspektive. In R. Fatke, W. Hornstein, C. Lüders \& M. Winkler (Hrsg.), Erziehung und sozialer Wandel. Brennpunkte sozialpädagogischer Forschung, Theoriebildung und Praxis (S. 17-38). Weinheim: Beltz.

Oberg, K. (2006). Cultural Shock: Adjustment to New Cultural Environments. Curare, 29(2+3), 142-146. (first published (1960): Practical Anthropology, 7(4), 177-182).

Peuckert, R. (2012). Familienformen im sozialen Wandel (8. Aufl.). Wiesbaden: Springer VS.

Pinderhughes, E.E., \& Rosenberg, K.F. (1990). Family-bonding with high risk placements: a therapy model that promotes the process of becoming a family. Journal of Children in Contemporary Society, 21(3-4), 209-230.

Pinderhughes, E. E., Matthews, J. A. K., \& Zhang, X. (2015). Research on adoptive families and their 21stcentury challenges. In S. Browning \& K. Pasley (Hrsg.), Contemporary families: translating research into practice (S. 14-34). New York: Routledge.

Reckwitz, A. (2015). Drei Versionen des Hybriden: Ethnische, kulturelle und soziale Hybriditäten. In T. Kron (Hrsg.), Hybride Sozialität - Soziale Hybridität (S. 187-200). Weilerswist: Velbrück.

Rosenthal, G., \& Loch, U. (2002). Das Narrative Interview. In D. Schaeffer \& G. Müller-Mundt (Hrsg.), Qualitative Gesundheits- und Pflegeforschung (S. 221-232). Bern: Huber.

Schütze, F. (1983). Biographieforschung und narratives Interview. Neue Praxis, 13(3), 283-293.

Smith, C. A. (1999). The encyclopedia of parenting theory and research. Chicago: Fitzroy Dearborn.

Smolin, D. M. (2005). The two faces of Intercountry adoption: the significance of the Indian adoption scandals. Seton Hall Law Review, 35, 403-493.

Strauss, A., \& Corbin, J. (1990). Basics of qualitative research. Grounded theory procedures and techniques. Newbury Park: SAGE.

Strübing, J. (2004). Grounded Theory. Zur sozialtheoretischen und epistemologischen Fundierung des Verfahrens der empirisch begründeten Theoriebildung. Wiesbaden: VS.

Suter, E.A., Baxter, L.A., Seurer, L.M., \& Thomas, L. J. (2014). Discursive constructions of the meaning of "family" in online narratives of foster adoptive parents. Communication Monographs, 81(1), 59-78.

Thieme, N. (2013). Kategorisierung in der Kinderund Jugendhilfe: Zur theoretischen und empirischen Erklärung eines Schlüsselbegriffs professionellen Handelns. Weinheim: Beltz Juventa.

Uhlendorff, U., Cinkl, S., \& Marthaler, T. (2008). Sozialpädagogische Familiendiagnosen. Deutungsmuster familiärer Belastungssituationen und erzieherischer Notlagen in der Jugendhilfe (Bd. 2). Weinheim: Juventa.

Vaskovics, L. A. (2016). Segmentierung und Multiplikation der Elternschaft und Kindschaft: ein Dilemma für die Rechtsregelung? Recht der Jugend und des Bildungswesens, 64, 194-209.

Wegar, K. (2004). Adoption, family ideology, and social stigma: bias in community attitudes, adoption research, and practice. Family Relations. Interdisciplinary Journal of applied Familiy Science, 49(4), 363-370.

Wind, L. H., Brooks, D., \& Barth, R. P. (2007). Influences of risk history and adoption preparation on postadoption services use in U.S. adoptions. Family Relations, 56(4), 378-389. 